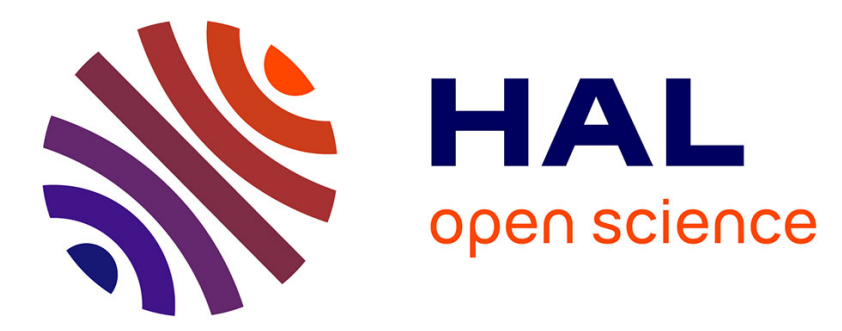

\title{
Aversion to risk of regret and preference for positively skewed risks
}

Christian Gollier

\section{To cite this version:}

Christian Gollier. Aversion to risk of regret and preference for positively skewed risks. Economic Theory, 2020, 70 (4), pp.913-941. 10.1007/s00199-018-1154-4 . hal-03142627

\section{HAL Id: hal-03142627 \\ https://hal.science/hal-03142627}

Submitted on 16 Feb 2021

HAL is a multi-disciplinary open access archive for the deposit and dissemination of scientific research documents, whether they are published or not. The documents may come from teaching and research institutions in France or abroad, or from public or private research centers.
L'archive ouverte pluridisciplinaire HAL, est destinée au dépôt et à la diffusion de documents scientifiques de niveau recherche, publiés ou non, émanant des établissements d'enseignement et de recherche français ou étrangers, des laboratoires publics ou privés. 


\title{
Aversion to risk of regret and preference for positively skewed risks*
}

\author{
Christian Gollier \\ Toulouse School of Economics, University of Toulouse-Capitole
}

September 14, 2016

\begin{abstract}
We assume that the ex-post utility of an agent facing a menu of lotteries depends upon the actual payoff together with its forgone best alternative, thereby allowing for the expost emotion of regret. An increase in the risk of regret is obtained when the actual payoff and its forgone best alternative are statistically less concordant in the sense of Tchen (1980). The aversion to any such risk of regret is thus equivalent to the supermodularity of the bivariate utility function. We show that more regret-risk-averse agents are more willing to choose the risky act in a one-risky-one-safe menu, in particular when the payoff of the risky choice is highly skewed. This is compatible with the "possibility effect" that is well documented in prospect theory. Symmetrically, we define the aversion to elationrisk that can prevail when the ex-post utility is alternatively sensitive to the forgone worst payoff. We show that elation-risk-seeking is compatible with the "certainty effect". We finally show that regret-risk-averse and elation-risk-seeking people behave as if they had rank-dependent utility preferences with an inverse-S shaped probability weighting function that reproduces estimates existing in the literature.
\end{abstract}

Keywords: Longshot bias, certainty effect, possibility effect, probability weighting, riskseeking, prospect theory, behavioral finance.

JEL codes: $\mathrm{D} 81$

${ }^{*}$ This paper was completed while the author was Wesley Clair Mitchell Visiting Research Professor at the Economics Department of Columbia University, whose hospitality is gratefully acknowledged. I want to thank Mohammed Abdellaoui, Keith Crocker, Glenn Harrison, Johannes Jaspersen, Chuck Mason, John Quiggin, Klaus Waelde, and Peter Wakker for particularly helpful comments. We also thank seminar participants at the Risk Theory Society meeting and at CESifo for their comments. The research leading to these results has received funding from the Chairs "Risk Markets and Value Creation", AMUNDI, and "Sustainable Finance and Responsible Investments" at TSE. 


\section{Introduction}

Regret and elation are emotional reactions to personal past acts. Regret is experienced when realizing that one would have been in a better situation, if only one would have decided differently. Symmetrically, elation is felt when realizing that we could have been in a worse situation. For example, one can extract some pleasure from the status quo if the alternative would have been a loss, as it happens when declining to bet on an event that does not materialize ex post. Regret can come from missed opportunities, such as failing to pass a medical test that would have revealed a cancer, or as not buying that asset whose market value has recently surged. It can also come from past actions yielding a bad outcome, such as lost gambles and unsuccessful investments. These emotions are mediated by a cognitive process known as counterfactual thinking involving the orbitofrontal cortex (Camille et al. (2004), Stalnaker et al. (2015)). If regret and elation affect utility ex post, it should not be a surprise that they do influence decisions ex ante. Since Gilovich and Medvec (1995), there is indeed overwhelming evidence in the psychology literature that people alter their choices in response to the anticipation of regret and elation (Zeelenberg and Pieters (2004), Zeelenberg and Pieters (2007)), with applications in marketing (Inman and McAlister (1994)), medicine (Brehaut et al. (2003), Chapman and Coups (2006)), insurance (Braun and Muermann (2004)), auctions (Filiz and Ozbay (2007)), and finance (Michenaud and Solnik (2008) and Muermann et al. (2006)) for example. ${ }^{1}$

Regret and elation are two symmetric emotions. Since Bell (1982) and Loomes and Sugden (1982), economists have explored the role of the anticipation of regret on optimal choices under uncertainty. ${ }^{2}$ Under the modern economic theory of regret that we reexamine in this paper, decision makers (DM) are assumed to maximize expected utility. But, when confronted with a non-trivial menu of lotteries, it is assumed that the DM's statewise utility $U$ depends not just on the state-specific payoff $x$ of the chosen lottery but also on the largest payoff $y$ that could have been obtained within the menu in that state. ${ }^{3}$ Observe that the distribution of the forgone best alternative is a function of the menu. This implies a potential intransitivity of the preference ordering. ${ }^{4}$ In the spirit of Loomes and Sugden (1982, 1987a,b), we measure the intensity of regret in any specific state by the difference between $y$ and $x$ that prevails in that state.

How does the anticipation of regret affect choice? The answer to this question obviously

\footnotetext{
${ }^{1}$ Zeelenberg and Pieters (2007) documents an exponentially increasing number of papers published on regret, starting around 1980 and culminating around 100 papers per year at the time of the publication of their paper.

${ }^{2}$ Bleichrodt and Wakker (2015) recently published a survey of regret theory on the occasion of the 125 th anniversary of the Economic Journal, recognizing the Loomes-Sugden's paper as one of the most pioneering publications of that journal.

${ }^{3}$ The original theory proposed by Bell $(1982,1983)$, Loomes and Sugden (1982, 1987a,b) and Loomes (1988)) was limited to menus with only two lotteries, and allowed for rejoice when the chosen lottery generated the largest payoff. In order to generalize the theory to menus containing more than two lotteries, and considering that people focus more on regret than on rejoice, Quiggin (1994) introduced an additional assumption into the model by claiming that statewise dominated alternatives should be irrelevant. This supports the theory of regret that has been used by economists since then, and that is used in the first part of this paper.

${ }^{4}$ For more on intransitivity with regret-sensitive preferences, see Bikhchandani and Segal (2011).
} 
depends upon the properties of the bivariate utility function. One key concept here is regret aversion. The problem is that the existing literature has not come up with a coherent and consensual definition of regret aversion. For example, Sarver (2008) defines regret aversion by the property that adding an ex ante suboptimal lottery in the choice menu makes the DM worse off. This is because this lottery can yield an ex post payoff that is larger than the payoff of the optimal lottery, thereby raising regret. Obviously, this definition of regret aversion is supported by the assumption that the utility function is decreasing in the forgone best alternative. Gee (2012) refers to this notion of regret aversion as first-order regret aversion. First-order regret aversion yields by definition a preference for a reduction of dominated options, and may justify regulations that reduce consumers' menu of choices (Huang et al. (2014)). In this paper, we introduce a new notion of aversion to risk of regret. $^{5}$ In line with the traditional way to define aversion, it would be consistent with the literature to say that someone is averse to the risk of regret if, everything else unchanged, she dislikes any mean-preserving spread in the distribution of the intensity of regret.

The primitive variables relevant for ex post utility is the actual payoff $x$ and the forgone best alternative $y$, from which one can derive the intensity of regret $y-x$. Ex ante, the expected utility of the DM depends upon the joint probability distribution of $(x, y)$. It is thus important to define the risk of regret from these pair of primitive variables. In this paper, we define an increase in risk of regret by a reduction in concordance between $x$ and $y$. The concept of comparative concordance was introduced in economics by Epstein and Tanny (1980) and Tchen (1980). Concordance is reduced by transferring some probability masses from the SW-NE corners to the NW-SE corners of any rectangle in the $(x, y)$-space, so that this change does not affect the marginal distributions of the two random variables. A reduction in concordance reduces covariance, but the opposite is not necessarily true. We show that a reduction in concordance between $x$ and $y$ increases the risk of $y-x$ in the sense of Rothschild and Stiglitz (1970), thereby justifying our reference to an increase in risk of regret. In other words, a reduction in concordance between the actual payoff and the forgone best alternative makes regret riskier without affecting the marginal distributions of the actual payoff and of the forgone best alternative. Our definition of aversion to risk of regret is that the DM dislikes any such reduction in concordance. For example, consider the following two menus described in Table 1 . Menu $M_{123}=\left\{x_{1}^{*}, x_{2}, x_{3}\right\}$ has three lotteries whose payoffs all depend upon the same draw of a fair coin. Let us contemplate the possibility to select lottery $x_{1}^{*}$ in this menu in which the best forgone alternative is 1 or 2 respectively in state $\mathrm{H}$ and $\mathrm{T}$. Because the actual payoff in these states are respectively 0 and 1 , regret takes value 1 with certainty. Let us alternatively contemplate the same choice $x_{1}^{*}$ in menu $M_{145}=\left\{x_{1}^{*}, x_{4}, x_{5}\right\}$. In that context, the statewise forgone best alternative are reversed, so that regret takes value 0 or 2 with equal probabilities. In short, the two risk contexts yields exactly the same marginal distributions for $x$ and $y$, but the second context has these variables less concordant. That yields an increase in the risk in regret. Under our definition, any regret-risk-averse DM should prefer the first menu over the second. The psychology and economics literature on the subject suggests that people are regret-averse in that sense, but much remains to be done to test this assumption. For example, Savage (1951), who introduced the notion of regret in

\footnotetext{
${ }^{5}$ Gee (2012) proposes a definition of second-order regret aversion that relies on the longshot bias. This is not intuitive. As we show in this paper, this merely substitutes an assumption by a result.
} 


\begin{tabular}{lcc}
\hline & $\mathrm{H}$ & $\mathrm{T}$ \\
\hline Lottery $x_{1}^{*}$ & 0 & 1 \\
Lottery $x_{2}$ & 1 & -1 \\
Lottery $x_{3}$ & -2 & 2 \\
\hline$y^{123}$ & 1 & 2 \\
\hline Regret & 1 & 1 \\
\hline
\end{tabular}

\begin{tabular}{|c|c|c|}
\hline & $\mathrm{H}$ & $\mathrm{T}$ \\
\hline Lottery $x_{1}^{*}$ & 0 & 1 \\
\hline Lottery $x_{4}$ & -1 & 1 \\
\hline Lottery $x_{5}$ & 2 & -2 \\
\hline$y^{145}$ & 2 & 1 \\
\hline Regret & 2 & 0 \\
\hline
\end{tabular}

Table 1: Regret-risk contexts in two menus of choices with a fair coin : $M_{123}=\left\{x_{1}^{*}, x_{2}, x_{3}\right\}$ and $M_{145}=\left\{x_{1}^{*}, x_{4}, x_{5}\right\}$.

economics, took the extreme view that a useful decision criterion under uncertainty is the "minmax regret" criterion in which this aversion to the risk of regret is infinite.

We show that a DM is averse to risk of regret if and only if $U$ is supermodular. We define the regret-risk premium associated to a marginal-preserving reduction in concordance between the actual payoff and its forgone best alternative as the sure reduction in actual outcome that compensates for it. We show that, in the small, the regret-risk premium equals the product of the increase in the covariance by an index of absolute aversion to risk of regret measured by $U_{x y} / U_{x}$. Our approach differs much from the economic literature in which most contributions assumed a specific functional form for the utility function, with $U(x, y)=u(x)-R(u(y)-u(x)){ }^{6}$ Although this specification is useful to intuitively separate the "choiceless/regretless/elationless" utility function $u$ from the penalty $R$ coming from the feeling of regret, we believe that it is too specific, with little benefit associated to this restriction.

An important contribution of this paper is to show that, in spite of the fact that our definition of regret-risk aversion is based on the attitude towards a second moment, this concept implies a preference for longshots, which is a bias towards a third moment. Let us consider a menu that contains a binary lottery and a sure payoff with the same mean. We show in this paper that in this context, moving from the safe choice to the risky one entails both an increase in the payoff-risk and a reduction in the regret-risk. This implies that the optimal choice is ambiguous under the combination of risk aversion and regret-risk aversion. Now, consider a mean-preserving reduction in the probability of gain that is compensated by an increase in that gain, making the risky bet a longshot. This change in distribution obviously increases the variance of the risky payoff. But we show that it reduces the risk of regret so much that the net effect is to make it more likely that the regret-risk-averse DM prefers the risky lottery over its safe alternative. To illustrate this result, consider a lottery in which there is a probability $p=10^{-6}$ to earn $10^{6}$ dollars against the paiement of one dollar. If one accepts the lottery, the distribution of regret is zero with probability $p$, and is 1 with the remaining probability. If one doesn't accept the lottery, the distribution of regret is $10^{6}-1$ dollars with probability $p$, and is zero otherwise. Clearly, accepting the lottery reduces the regret risk, but it raises the payoff risk. And obviously, the large skewness of the

\footnotetext{
${ }^{6}$ Quiggin (1994) is an exception.
} 
lottery payoff makes this argument more convincing. In the case of racetrack betting, this generates a preference for longshots, a bias that is well documented by Ali (1977), Thaler and Ziemba (1988) and Jullien and Salanié (2000). ${ }^{7}$ Notice however that the preference for positively skewed lotteries can potentially be explained by assuming that people are prudent in the classical expected utility model (Kimball (1990)).

In decision theory, the longshot bias is called the "possibility effect" in which people tend to overestimate the probability of low-probability high-payoff event (Wakker (2010)). In the Rank-Dependent Utility (RDU) model, the possibility effect takes the form of a steep relation between the objective probability $p$ of the high payoff and its "weight" $w$ used to measure wellbeing ex ante, in the neighborhood of $p=0$. However, regret-risk aversion cannot explain another classical finding of experimental studies, namely the "certainty effect", in which people have an incremental risk aversion towards risk yielding a low probability of the lower payoff. In the binary RDU model, this certainty effect is reflected in a steep relation between $p$ and $w$ in the neighborhood of $p=1$. Combining the possibility effect and the certainty effect suggests a concave-convex (or inverse-S shaped) probability weighting function $w{ }^{8}$ We will show that regret-risk aversion can explain the concave-convex probability weighting $w$, but it fails to explain the certainty effect. In fact, the problem is that regret-risk aversion implies an optimistic RDU transformation, with $w(p) \geq p$ for all $p$.

As explained earlier, another possible counterfactual emotion is elation or rejoicing, a sensation felt when the actual payoff is larger than the worst alternative outcome. In Section 7, we develop a dual theory of elation-risk seeking that basically duplicates our theory of regret-risk aversion in which the forgone best alternative is replaced by the forgone worst alternative in the utility function. We show that elation-risk aversion yields a binary RDU probability weighting in the domain of pessimism $(w \leq p)$, and that it can explain the certainty effect. We also show that combining regret-risk aversion and elation-risk seeking in the expected utility model can generate a binary RDU probability weighting $w$ that almost exactly duplicates the inverse-S shape one proposed by Tversky and Kahneman (1992), for example.

Section 2 is devoted to the definitions and the characterization of the risk of regret and of regret-risk aversion. We also show there the link between the risk of regret and the reduction in concordance between the payoff and the forgone best alternative. In Section 3, we define the regret-risk premium and we derive an index of regret-risk aversion that is consistent with these definitions. We derive an approximation à la Arrow-Pratt of the former by using the latter. In Section 4, we examine the role of regret-risk aversion in the risk attitude towards menus that contain a binary lottery and its mean (or certainty equivalent), and we characterize the link between regret-risk aversion and the preference for positively skewed

\footnotetext{
${ }^{7}$ See also Golec and Tamarkin (1998), Garrett and Sobel (1999), Harvey and Siddique (2000), Bhattacharya and Garrett (2008), and Eichner and Wagener (2011). By showing that adding low-probability macroeconomic catastrophes into the beliefs of the representative agent can explain the equity premium puzzle, Barro (2006, 2009 ) is in line with the idea that investors particularly dislike negatively skewed returns.

${ }^{8}$ See for example Tversky and Kahneman (1992), Wu and Gonzalez (1996), Abdellaoui (2000), Abdellaoui et al. (2010). Wakker (2010) gives some dozens of references confirming the inverse-S probability weighting function, together with some dissenting studies.
} 
lotteries. We generalize these results in Section 5 for non-binary lotteries. Section 6 links our findings to the binary RDU model, whereas we present a dual theory of elation-risk seeking in Section 7. Section 8 is devoted to a short analysis of the two-asset portfolio problem.

\section{Definition and characterization of the aversion to regret-risk}

The uncertainty is described by a set of $S$ possible states of nature, indexed $s=1, \ldots, S$. There is an objective probability distribution of the states given by vector $\left(p_{1}, \ldots, p_{S}\right)$ in the simplex of $\mathbb{R}^{S}$. In this paper, the expectation operator is always related to the state of nature, in the sense that $E f=\sum_{s=1}^{S} p_{s} f(s)$ for all $f: S \rightarrow \mathbb{R}$. A lottery (or an act) is defined by a function from $S$ to $\mathbb{R}$ that specifies the final payoff $x(s)$ in each state $s$. The choice problem is characterized by a menu $M=\left\{x_{\theta}: S \rightarrow \mathbb{R} \mid \theta \in \Theta\right\}$ of lotteries indexed by $\theta$ in some index set $\Theta$. In the spirit of Bell $(1982,1983)$, Loomes and Sugden $(1982,1987 a, b)$ and following Quiggin (1994), we assume that the decision-maker is sensitive to regret in the sense that her utility $U$ in any state $s$ is a function of two variables: (1) the actual payoff $x(s)$ of the chosen lottery, and (2) the maximal consumption $y^{M}(s)$ that could have been attained in that state if another feasible choice had been made at the beginning of the period: $y^{M}(s)=\max _{\theta \in \Theta} x_{\theta}(s)$ for all $s \in S$. To keep the analysis simple, we assume that $\Theta$ is finite, so that such a function $y^{M}$ exists. The choice problem ex ante takes thus the general form of a choice among the different lotteries existing in menu $M:^{9}$

$$
\max _{\theta \in \Theta} E U\left(x_{\theta}, y^{M}\right)
$$

We assume that the decision-maker always prefers to consume more to less, and is averse to risk on actual consumption. More precisely, $U$ is increasing in $x$, which means that any first-order stochastic improvement in the conditional distributions $x \mid y=b$ increases welfare, for any $b \in \mathbb{R}$. Similarly, $U$ is concave in $x$, which means that any increase in risk of $x \mid y=b$ reduces welfare. We now define the notion of regret aversion. To do this, we need to define a preference order over menus. We say that a menu $M$ is preferred to another menu $N$ if and only if the expected utility generated by the optimal choice in menu $M$ is larger than the optimal choice in menu $N$. Let $x_{1}$ and $x_{2}$ denote two lotteries. Sarver (2008) and Gee (2012) define regret aversion as follows: ${ }^{10}$ If menu $M_{2}=\left\{x_{2}\right\}$ is preferred to menu $M_{1}=\left\{x_{1}\right\}$, then adding lottery $x_{1}$ in a menu that already contains $x_{2}$ cannot make that menu more attractive. In particular, this means that

$$
E U\left(x_{2}, x_{2}\right) \geq E U\left(x_{1}, x_{1}\right) \Rightarrow E U\left(x_{2}, x_{2}\right) \geq E U\left(x_{2}, y\right),
$$

with $y(s)=\max \left\{x_{1}(s), x_{2}(s)\right\}$ for all $s \in S$. The intuition is that adding an ex-ante dominated lottery can potentially increase the best alternative outcome in some states, thereby raising the negative feeling of regret in these states. It is obvious that regret aversion holds if and only if $U$ is decreasing in $y$. It is useful to measure the intensity of regret $r(s)$ in any

\footnotetext{
${ }^{9}$ This formulation encompasses a wide variety of choice problems, such as the problem of choosing a portfolio of assets, or of selecting the best insurance coverage of an insurable risk.

${ }^{10}$ In fact, Sarver (2008) calls this "regret", but this is clearly a concept of aversion to regret. Gee (2012) refers to this notion as "first-order regret aversion".
} 


\begin{tabular}{llllllll}
\hline \hline Ball number & 0 & 1 & $\ldots$ & 49 & 50 & $\ldots$ & 99 \\
\hline Lottery $x_{1}^{*}$ & 0 & 1 & $\ldots$ & 49 & 50 & $\ldots$ & 99 \\
Lottery $x_{0}$ & 100 & 0 & $\ldots$ & 0 & 0 & $\ldots$ & 0 \\
\hline$y^{01}=\max \left\{x_{1}^{*}, x_{0}\right\}$ & 100 & 1 & $\ldots$ & 49 & 50 & $\ldots$ & 99 \\
\hline Regret & 100 & 0 & $\ldots$ & 0 & 0 & $\ldots$ & 0 \\
\hline \hline
\end{tabular}

Table 2: Regret-risk context in a menu $M_{01}$ with an urn containing 100 balls numbered from 0 to 99 .

\begin{tabular}{lccccccc}
\hline \hline Ball number & 0 & 1 & $\ldots$ & 49 & 50 & $\ldots$ & 99 \\
\hline Lottery $x_{1}^{*}$ & 0 & 1 & $\ldots$ & 49 & 50 & $\ldots$ & 99 \\
Lottery $x_{2}$ & 1 & 2 & $\ldots$ & 50 & 0 & $\ldots$ & 0 \\
Lottery $x_{3}$ & 0 & 0 & $\ldots$ & 0 & 51 & $\ldots$ & 100 \\
\hline$y^{123}=\max \left\{x_{1}^{*}, x_{2}, x_{3}\right\}$ & 1 & 2 & $\ldots$ & 50 & 51 & $\ldots$ & 100 \\
\hline Regret & 1 & 1 & $\ldots$ & 1 & 1 & $\ldots$ & 1 \\
\hline \hline
\end{tabular}

Table 3: Regret-risk context in a menu $M_{123}$ with an urn containing 100 balls numbered from 0 to 99 .

specific state $s$ by the difference between the forgone best alternative $y(s)$ and the actual payoff $x(s): r(s)=y(s)-x(s)$. It is menu-specific. By definition, the intensity of regret is non-negative. Regret aversion means that the agent dislikes any increase in state regret. Given the fact that $y$ can only increase when enlarging the menu of choices, regret aversion is a also a preference for reducing the choice opportunity set containing the optimal solution (Sarver (2008)).

By contrast, our results rely on another concept that we call Aversion to Risk of Regret (ARR). To illustrate, let us consider an urn that contains 100 balls numbered from 0 to 99 . A ball is randomly extracted from the urn. Lottery $x_{0}$ yields a payoff of 100 if ball numbered 0 is extracted from the urn, and a payoff of 0 otherwise. Lottery $x_{1}^{*}$ yields a payoff equaling the number of the ball extracted from the urn. Suppose that the agent prefers lottery $x_{1}^{*}$ in menu $M_{01}=\left\{x_{0}, x_{1}^{*}\right\}$, as described in Table 2. Observe that the agent faces regret only if ball 0 is obtained. In other words, the intensity of regret $r$ is 100 with probability $1 \%$, and is 0 otherwise.

Let us contemplate alternatively menu $M_{123}=\left\{x_{1}^{*}, x_{2}, x_{3}\right\}$ described in Table 3 . Lottery $x_{2}$ yields a payoff of $s+1$ if the number of the ball is $s$ if $s$ is less or equal to 49, and 0 otherwise. Lottery $x_{3}$ yields a payoff of $s+1$ if the number of the ball is $s$ if $s$ is larger or equal to 50, and 0 otherwise. Suppose that the agent also prefer lottery $x_{1}^{*}$ in menu $M_{123}$. But the agent will always face regret from not having selected lottery $x_{2}$ (if $s \leq 49$ ) or lottery $x_{3}$ (if $s \geq 50$ ) ex post, yielding an intensity of regret $r$ equaling unity with certainty. 
Let us now compare the risk outcomes of the two menus $M_{01}$ and $M_{123}$ in more detail. Because $x_{1}^{*}$ is always preferred, the marginal distribution of the final payoff is the same in the two menus. Observe also that the marginal distributions of the best alternative payoff $y$ are also identical in the two menus. More specifically, the marginal distribution of $y$ is uniform over the set $\{1,2, \ldots, 100\}$ in both menus. Thus, in terms of the marginal distributions of $x$ and $y$, the two menus are identical. However, the distributions of regret $r$ are different. Their expectations are equal, but menu $M_{01}$ generates an increase in the risk of regret compared to menu $M_{123}$. Thus, a regret-risk-averse agent should prefer menu $M_{123}$ over menu $M_{01}$.

Because the intensity of regret is measured by the difference between $x$ and $y$, risk on regret increases when these two random variables are less statistically concordant, a concept developed by Tchen (1980) and Epstein and Tanny (1980) in decision theory, and by Atkinson and Bourguignon (1982) in welfare economics. To show this, let us compare two risk contexts characterized respectively by $\left(x_{1}, y_{1}\right)$ and $\left(x_{2}, y_{2}\right)$. Let $F_{i}: \mathbb{R}^{2} \rightarrow \mathbb{R}$ denote the bivariate distribution function associated to context $i, i=1,2$. Suppose that $F_{2}$ is obtained from $F_{1}$ through a Marginal-Preserving Reduction in Concordance (MPRC). A MPRC is based on two transfers of probability masses among four realizations of $(x, y)$, with $x \in\{a, A\}, a<A$ and $y \in\{b, B\}$, with $b<B$, as shown in Figure 1. First, probability mass $\varepsilon$ in the neighborhood of $(a, b)$ is transferred upwards in the neighborhood of $(a, B)$. Second, probability mass $\varepsilon$ in the neighborhood of $(A, B)$ is transferred downwards in the neighborhood of $(A, b)$. Observe that this does not change the marginal distributions of $x$ and $y$. A numerical application of such a MPRC is described by moving from menu $M_{123}$ to menu $M_{145}$ in Table 1. MarginalPreserving Increases in Concordance (MPIC) are defined symmetrically.

Any MPRC reduces the correlation between $x$ and $y$, and it yields a mean-preserving spread in the distribution of the intensity $r=y-x$ of regret in the sense of Rothschild and Stiglitz (1970), as claimed in the following proposition.

Proposition 1. Any marginal-preserving reduction in concordance in $(x, y)$ yields a meanpreserving spread in regret $r=y-x$.

Proof. A mean-preserving spread in $r$ is obtained by defining an interval $I$ in the support of $r$ from which some probability mass is extracted to be transferred outside $I$, preserving the mean of $r$. Define $r_{\min }=\min \{B-A, b-a\}$ and $r_{\max }=\max \{B-A, b-a\}$. Let us consider interval $I=\left[r_{\min }, r_{\max }\right]$. The MPRC described above transfers a probability mass $\epsilon$ in the distribution of regret $r=y-x$ from $I$ to $B-a>r_{\text {max }}$, and another probability mass $\epsilon$ from $I$ to $A-b<r_{\text {min }}$. Moreover, this change in the distribution of regret preserves the mean. This is because the MPRC preserves the mean of $x$ and $y$, thereby preserving the mean of $r=y-x$.

More generally, $F_{2}$ is said to be less concordant than $F_{1}$ if and only $F_{2}$ is obtained from $F_{1}$ through a sequence of MPRCs. Tchen (1980) and Epstein and Tanny (1980) have shown that $F_{2}$ is less concordant than $F_{1}$ if and only if they have the same marginal distributions and

$$
\forall(a, b) \in[\underline{x}, \bar{x}] \times[\underline{y}, \bar{y}] \subset \mathbb{R}^{2}: F_{2}(a, b) \leq F_{1}(a, b) .
$$




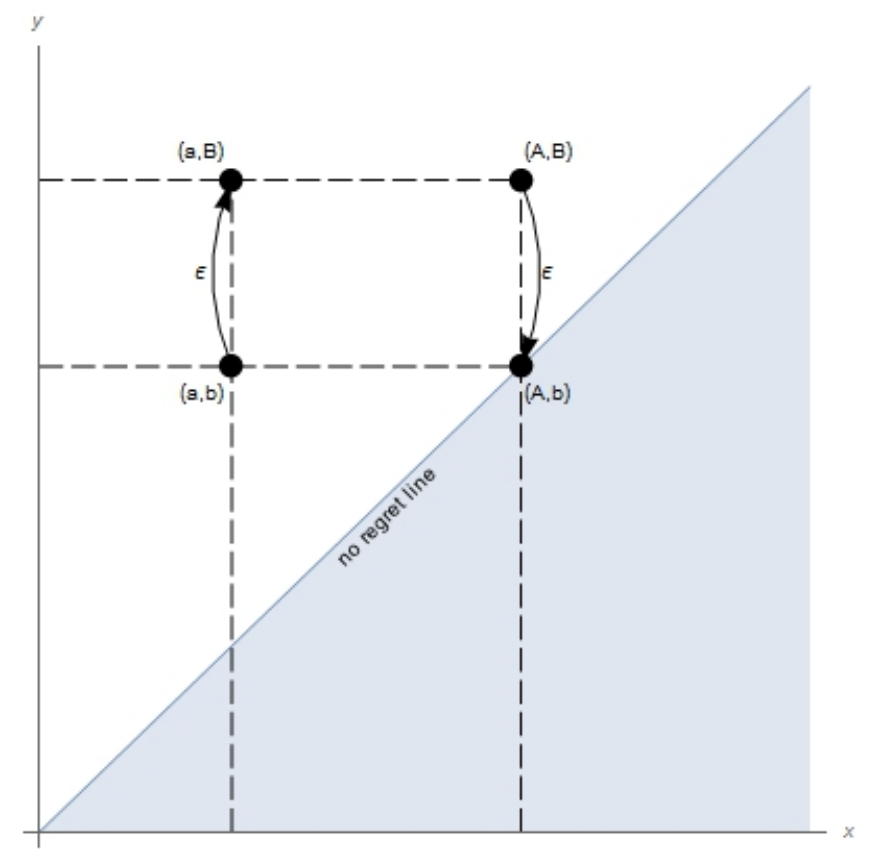

Figure 1: Example of a marginal-preserving reduction in concordance (MPRC).

Remember that because a reduction in concordance has no effect on the marginal distributions of $x$ and $y$, it does not affect the riskiness of the final payoff and of the best alternative payoff. But it increases the risk of regret since the sequence of MPRCs that generates it yields a sequence of mean-preserving spreads in the distribution of regret, i.e., a Rothschild-Stiglitz increase in risk of regret.

Definition 1. Let $F_{1}$ and $F_{2}$ be two cumulative distribution functions from $[\underline{x}, \bar{x}] \times[\underline{y}, \bar{y}] \subset$ $\mathbb{R}^{2} \rightarrow \mathbb{R}$ having the same marginal distributions. $F_{2}$ exhibits more risk of regret than $F_{1}$ if and only if $F_{2}$ is less concordant than $F_{1}$, i.e., if and only if condition (3) is satisfied.

This justifies the following definition of ARR.

Definition 2. $U$ is averse to risk of regret if any increase in risk of regret reduces expected utility.

It is easy to show that in the numerical illustration described in tables 2 and 3 , menu $M_{01}$ yields a risk context $(x, y)$ that is less concordant than menu $M_{123}$, which implies an increase in risk of regret. ${ }^{11}$ All agents that are averse to risk of regret should therefore prefer menu $M_{123}$ over menu $M_{01}$. Observe now that the MPRC described in Figure 1 reduces expected utility $E U(x, y)$ if and only if for all $a \leq A$ and $b \leq B$,

$$
U(a, b)+U(A, B) \geq U(a, B)+U(A, b) .
$$

\footnotetext{
${ }^{11}$ This reduction of concordance can be obtained through a sequence of 100 MPRCs. The first is to move the 0.01 probability mass at $(0,100)$ downward to $(0,99)$, and to compensate this by an upward move of the same probability mass from $(99,99)$ upward to $(99,100)$.
} 
By definition, this is true if and only if $u$ is supermodular. When $U$ is twice differentiable, this means that $U_{x}$ is increasing in $y$, or $U_{x y} \geq 0$. This yields the following result, which is an application of Epstein and Tanny (1980). This result implies in particular that an increase in risk of regret reduces the covariance between $x$ and $y$.

Theorem 1. $U$ is averse to risk of regret if and only if $U$ is supermodular.

Of course, by symmetry, regret-seekers have a submodular utility function. Thus, the aversion to regret corresponds to $U$ being decreasing in $y$, whereas the aversion to risk of regret corresponds to $U_{x}$ being increasing in $y$. Because $y$ is a bad under regret aversion, the aversion to risk of regret can also be interpreted as a preference for mixing good $(x)$ with bad $(y)$, a common property of individual preferences first suggested by Eeckhoudt and Schlesinger (2006). ${ }^{12}$

\section{Measure of aversion to risk of regret}

It is natural to define the premium associated to an increase in risk of regret by the sure reduction in consumption that has the same effect on expected utility.

Definition 3. Consider an increase in risk of regret from $F_{1}$ to $F_{2}$. The associated regret risk premium $\pi$ is defined as follows:

$$
\iint U(x-\pi, y) d F_{1}(x, y)=\iint U(x, y) d F_{2}(x, y)
$$

Because $U$ is increasing in its first argument, the regret-risk premium is non-negative under regret-risk aversion. Condition (5) can be rewritten as

$$
\iint[U(x, y)-U(x-\pi, y)] d F_{1}(x, y)=\iint U(x, y) d\left(F_{1}(x, y)-F_{2}(x, y)\right)
$$

Suppose that the increase in risk of regret is limited to a sequence of small MPRCs in the neighborhood of $(a, b)$. The left-hand side of equation (6) can then be approximated by $U_{x}(a, b) \pi$. Using a second-order Taylor approximation, and taking advantage of the fact that the marginals are unaffected by the change in distribution, we also have that

$$
\begin{aligned}
\iint U(x, y) & d\left(F_{1}(x, y)-F_{2}(x, y)\right) \\
& \approx U_{x y}(a, b) \iint(x-a)(y-b) d\left(F_{1}(x, y)-F_{2}(x, y)\right) \\
& =U_{x y}(a, b)\left[\operatorname{cov}_{1}(x, y)-\operatorname{cov}_{2}(x, y)\right] \\
& =U_{x y}(a, b) \triangle \operatorname{cov}(x, y)
\end{aligned}
$$

where $\operatorname{cov}_{i}(x, y)$ is the covariance between $x$ and $y$ under distribution $F_{i}$, and $\triangle \operatorname{cov}(x, y)$ is the reduction in covariance in $(x, y)$ that is associated to this increase in risk of regret. Equation (6) then implies that

$$
\pi \approx \frac{U_{x y}(a, b)}{U_{x}(a, b)} \triangle \operatorname{cov}(x, y)
$$

\footnotetext{
${ }^{12}$ These authors examined the special case of a univariate utility function. Eeckhoudt et al. (2007) extended this analysis to the case of a multivariate utility function.
} 
This justifies the following definition of an index of absolute aversion to risk of regret (AARR), which parallels the standard Arrow-Pratt definition of absolute risk aversion. Gollier and Salanié (2012) used this definition to characterize optimal portfolios and asset prices in an Arrow-Debreu economy with regret-risk-averse agents.

Definition 4. We define the indexes of absolute risk aversion $\alpha$ and of absolute regret-risk aversion $\rho$ as follows:

$$
\alpha(a, b)=-\frac{U_{x x}(a, b)}{U_{x}(a, b)}, \text { and } \rho(a, b)=\frac{U_{x y}(a, b)}{U_{x}(a, b)} .
$$

When the increase in risk of regret is localized in the neighborhood of $(a, b), \rho(a, b)$ is the certainty equivalent reduction in consumption corresponding to a unit reduction in the covariance between the actual payoff and the forgone best alternative. For example, if $(A, B)$ is in the neighborhood of $(a, b)$ in Figure 1, the corresponding MPRC has a regret risk premium that can be approximated by this equation:

$$
\pi \approx \Delta p \Delta x \Delta y \rho(a, b),
$$

with $\Delta p=\varepsilon, \Delta x=A-a$ and $\Delta y=B-b$.

Various specifications of the bivariate utility function $U$ exist in the literature. Bell (1982) proposed to use a function $U(x, y)=u(x)-R(u(y)-u(x))$, where $u$ is an increasing and concave "choiceless" utility function, and $R$ is a regret penalty function. ${ }^{13}$ Several authors, such as Braun and Muermann (2004), Muermann et al. (2006), Sarver (2008), Michenaud and Solnik (2008), and Gee (2012) for example, have followed this tradition. Under this specification, the index of regret-risk aversion equals $\rho(a, b)=u^{\prime}(b) R^{\prime \prime} /\left(1+R^{\prime}\right)$ where the derivatives of $R$ are evaluated at $u(b)-u(a)$. Notice that disentangling regret-risk aversion from risk aversion is only partial in this model, since the index of risk aversion $\alpha$ obviously depends upon the shape of $R$.

Savage (1951) proposed the decision criterion in which the DM minimizes the maximum statewise regret $r(x, y)=y-x$. This can be interpreted as an extreme version of our general model with $U(x, y)=u(r(x, y))$ and $u(r)=-A^{-1} \exp (A r)$ for $A \in \mathbb{R}^{+}$. This implies indexes $\alpha(a, b)=\rho(a, b)=A$. Let us define the certainty equivalent regret $R$ as $u(R)=E u(r(x, y))$. This is equivalent to $R$ equaling $A^{-1} \ln E \exp (A r)$, which is the cumulant-generating function of random variable $r$. As is well-known, when $A$ tends to infinity, $R$ tends to the maximum statewise regret. Because $u$ is decreasing in $R$, maximizing $u(R)=E U(x, y)$ is equivalent to minimizing $R$. So this specification of our general model leads to the minmax regret criterion that has played an historical role in the development of decision theory during the last century.

Finally, in the spirit of this exponential specification, we propose a multiplicative formulation with $U(x, y)=u(x) v(y)$, with $u^{\prime} \geq 0, u^{\prime \prime} \leq 0, v \geq 0$. Under this specification, the agent is averse to risk of regret if and only if $v$ is increasing: $v^{\prime} \geq 0$. The index of regret-risk aversion is $\rho(a, b)=v^{\prime}(y) / v(y)$, whereas the index of risk aversion is $\alpha(a, b)=-u^{\prime \prime}(a) / u^{\prime}(a)$. This specification allows for a full separation of risk and regret-risk attitudes in the small.

\footnotetext{
${ }^{13}$ Loomes and Sugden $(1982,1987 b)$ considered the special case of this specification with $u(x)=x$.
} 


\section{The one-risky-one-safe-lottery menu with two states: Pref- erence for longshots}

In this section, we explore the link between the attitude towards skewed risk and the aversion to risk of regret as defined in the previous section. Although we defined ARR strictly in relation to mean-preserving spreads in the intensity of regret, we hereafter show that ARR generates a form of preference in favor of skewed risks in consumption.

To do this, we examine simple menus $M=\left\{x_{1}, x_{2}\right\}$ that contain two lotteries. We further assume in this section that lottery $x_{2}$ is safe as it generates a payoff equaling the mean of $x_{1}$ with certainty. Without loss of generality, we assume that $E x_{1}=0$. We examine the conditions under which, in spite of her risk aversion, the decision-maker wants to select the risky lottery over its mean in this menu. In this problem, the agent faces two risks of regret depending upon her decision. First, if she takes the risky lottery, she will feel regret if she makes a loss on this gamble. Second, if she does not take the risk, she will feel regret if the payoff of the lottery is positive. If the risky lottery yields a large payoff with a small probability, the risk of regret is larger in this second scenario than in the first. If the skewness of the risky lottery is large enough, the effect of aversion to risk of regret may dominate the effect of risk aversion to induce the decision-maker to prefer the risky lottery in menu $M$. In this section, we consider the special case in which the risky lottery $x_{1}$ of this menu is binary, with payoffs $K$ and $-k$ respectively with probability $p$ and $1-p$, in the spirit of March (1996). We assume that $K=k(1-p) / p$ in order to have $E x_{1}=0$. This lottery can be interpreted as betting $k>0$ on a horse whose probability to win the race is $p$, under an actuarially fair pricing. Without loss of generality, let's assume at this stage $k=1$. Obviously, a reduction in $p$ raises the skewness of $x_{1}$. Because the alternative choice in menu $M$ is $x_{2}={ }_{p} 0$, the distribution of forgone best alternative $y$ associated to this menu is characterized by $y=0$ if $x_{1}=-k$ and $y=K$ if $x_{1}=K$.

\subsection{Selecting the risky lottery yields an increase in payoff-risk and a re- duction in regret-risk}

In Figure 2, we drew in red the distribution of the risk context $\left(x_{2}, y^{M}\right)$ if the safe lottery $x_{2}$ is selected. It takes value $(0,0)$ and $(0, K)$ respectively with probability $1-p$ and $p$. We also drew in blue the distribution of $\left(x_{1}, y^{M}\right)$ when the risky lottery is selected.

We hereafter show how to transfer probability masses to transform the risk context where the safe lottery $x_{2}$ is selected in menu $M$ into the one in which the risky lottery $x_{1}$ is selected, i.e., when moving from red to blue. A sequence of three transfers of probability masses will generate this transformation, two yielding a mean-preserving spread (MPS) in the distribution of $x$ conditional to $y$, and one yielding a marginal-preserving increase in concordance (MPIC):

- A MPS in $x$ conditional to $y=0$ : The probability mass $1-p$ at $(0,0)$ is split into $(1-p)^{2}$ and $p(1-p)$. These masses are transferred horizontally respectively to $(-k, 0)$ and to $(K, 0)$. 


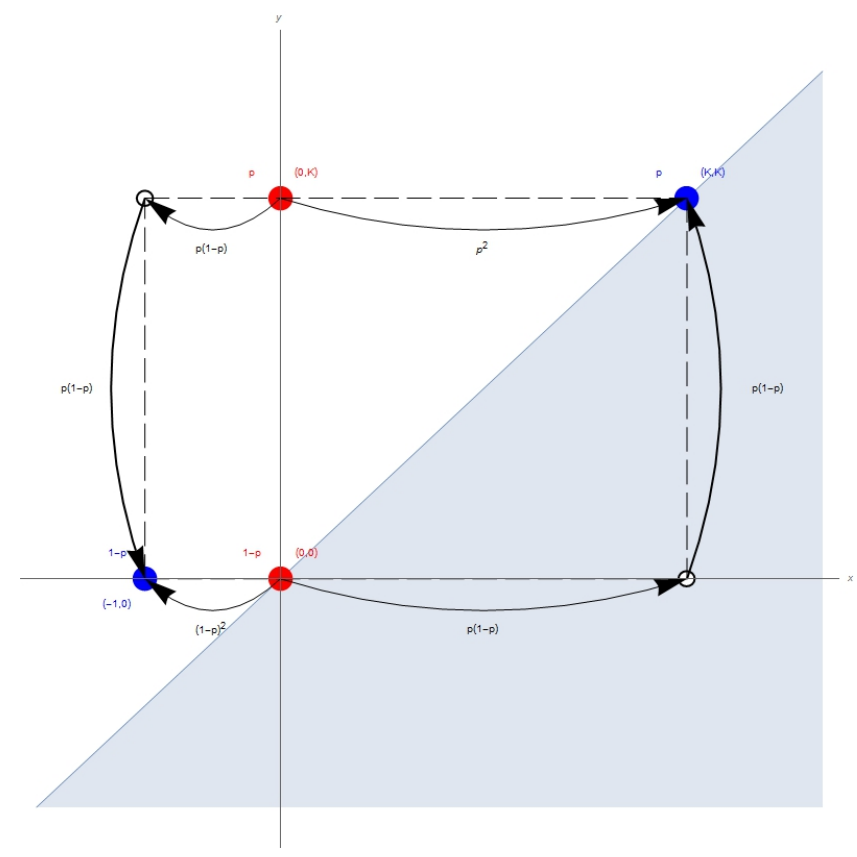

Figure 2: Transfers of probability masses in the $x, y$-space in the context of a menu $M=$ $\left\{x_{1} \sim(K, p ;-1,1-p), x_{2}={ }_{p} 0\right\}$.

- A MPS in $x$ conditional to $y=K$ : The probability mass $p$ at $(0, K)$ is split into $p(1-p)$ and $p^{2}$. These masses are transferred horizontally respectively to $(-k, K)$ and to $(K, K)$.

- A MPIC: The two probability masses $p(1-p)$ now at $(-k, K)$ and $(K, 0)$ are transferred vertically respectively at $(-k, 0)$ and $(K, K)$.

This sequence of three transfers of probability masses are represented in Figure 2. They transform the risk environment $\left(x_{1}, y^{M}\right)$ into $\left(x_{2}, y^{M}\right)$. Because the agent is risk-averse in $x\left(U_{x x} \leq 0\right)$, the two mean-preserving spreads in $x \mid y$ reduce expected utility. But the marginal-preserving increase in concordance reduces the risk of regret. Under the regret risk aversion $\left(U_{x y} \geq 0\right)$, the MPIC involved in the transformation of the risk context $(x, y)$ from the safe lottery to the risky one raises expected utility, thereby making the optimal choice in $M$ ambiguous.

\subsection{The case of small risk}

This ambiguity can be removed if we examine the special case of $x_{1}$ being a small binary risk, as shown in our next proposition.

Proposition 2. Consider a menu $M=\left\{x_{1}, x_{2}\right\}$ with $x_{1} \sim(k(1-p) / p, p ;-k, 1-p)$ and $x_{2}={ }_{p} 0$. In this menu, switching from the safe lottery $x_{2}$ to the risky one $x_{1}$ yields a MPS in $x \mid y=x_{1}$, a MPS in $x \mid y=x_{2}$, and a MPIC. The corresponding increase in expected utility equals 


$$
E U\left(x_{1}, y^{M}\right)-E U\left(0, y^{M}\right)=k^{2} \frac{1-p}{p} U_{x}(0,0)\left[(1-p) \rho_{0}-0.5 \alpha_{0}\right]+O\left(k^{3}\right),
$$

where $\rho_{0}=\rho(0,0)$ and $\alpha_{0}=\alpha(0,0)$ are the aversion to respectively regret-risk and risk, as defined by equation (9).

Proof. We rewrite the left-hand side of equation (11) as follows:

$$
f(k)=p U\left(k \frac{1-p}{p}, k \frac{1-p}{p}\right)+(1-p) U(-k, 0)-p U\left(0, k \frac{1-p}{p}\right)-(1-p) U(0,0) .
$$

It is easy to check that $f(0)=f^{\prime}(0)=0$ and that

$$
f^{\prime \prime}(0)=U_{x x}(0,0) \frac{1-p}{p}+2 U_{x y}(0,0) \frac{(1-p)^{2}}{p}=\frac{1-p}{p} U_{x}(0,0)\left[2(1-p) \rho_{0}-\alpha_{0}\right] .
$$

This implies that

$$
f(k)=\frac{1}{2} k^{2} f^{\prime \prime}(0)+O\left(k^{3}\right)=k^{2} \operatorname{Var}\left(x_{1}\right) U_{x}(0,0)\left[(1-p) \rho_{0}-0.5 \alpha_{0}\right]+O\left(k^{3}\right) .
$$

This concludes the proof.

Suppose now that bet $k$ is close to zero. In that context, the risk premium associated to $x_{1}$ is approximately to its Arrow-Pratt approximation $0.5 \alpha_{0} E x_{1}^{2}$, with $E x_{1}^{2}=k^{2}(1-p) / p$ and $\alpha_{0}=\alpha(0,0)$. This measures the certainty equivalent loss in $x$ associated to the sequence of the two MPS described above. Similarly, following equation (10), the (negative) regret risk premium associated to the MPIC can be approximated by $-k^{2}(1-p)^{2} \rho_{0} / p$, with $\rho_{0}=\rho(0,0)$, since $\Delta p=-p(1-p), \Delta x=k / p$ and $\Delta y=k(1-p) / p$. This provides an intuition to Proposition 2.

Observe that the MPIC necessary to transform the risk context $\left(x_{2}, y^{M}\right)$ into $\left(x_{1}, y^{M}\right)$ reduces the covariance between $x$ and $y$ by $(1-p)^{2} / p$, whereas the increase in variance in the final payoff equals $(1-p) / p$. This means that

$$
\Delta \operatorname{cov}(x, y)=(1-p) \operatorname{Var}\left(x_{1}\right) .
$$

Because the negative regret risk premium is approximately proportional to $\Delta \operatorname{cov}(x, y)$ whereas the positive risk premium is approximately proportional to $\operatorname{Var}\left(x_{1}\right)$, we can conclude that the relative effect of the aversion to risk of regret is decreasing in $p$. In other words, our definition of ARR is compatible with a preference for longshots. This is formalized in the following corollary, which is a direct consequence of equation (11).

Corollary 1. Under the assumptions of Proposition 2, and assuming that the size $k$ of the bet is small, then

- the safe lottery is always preferred if and only if the regret-risk aversion is smaller than half the risk aversion, i.e., iff $\rho(0,0) \leq 0.5 \alpha(0,0)$; 
- a mean-preserving reduction in the probability of success $p$ makes the risky choice more desirable. Technically, if the risky lottery $x_{1} \sim(k(1-p) / p, p ;-k, 1-p)$ is preferred to 0 in menu $M=\left\{x_{1}, 0\right\}$, then for all $p^{\prime} \leq p$, the risky lottery $x_{1}^{\prime} \sim\left(k\left(1-p^{\prime}\right) / p^{\prime}, p^{\prime} ;-k, 1-\right.$ $\left.p^{\prime}\right)$ is preferred to 0 in menu $M=\left\{x_{1}^{\prime}, 0\right\}$.

The first result in this corollary states that there is a strictly positive lower bound (equaling $0.5 \alpha_{0}$ ) for the regret risk aversion $\rho_{0}$ below which the risky choice in $M$ can never be optimal. This lower bound is obtained from equation (11) by pushing the skewness of the risky lottery to its extreme, with $p \rightarrow 0$ and $K / k \rightarrow \infty$. The second result illustrates a preference for longshots when $\rho_{0}>0.5 \alpha_{0}$. In fact, under our assumption of an actuarially fair pricing, betting on a horse whose probability to win is $p$ is optimal if and only if $p$ is smaller than $1-0.5 \alpha_{0} / \rho_{0}$.

\section{The general case of the one-risky-one-safe-lottery menu}

In this section, we relax the assumption that the risky lottery has only two atoms. In other words, we characterize the choice of regret-risk-averse agents who face a menu that contains an arbitrary lottery and a safe bet. Since the impact of a change in the payoff of the safe bet has a trivial consequence on the choice, we hereafter assume that the two choices yield the same expected payoff. The generalization presented in this section is derived from the following lemma, whose proof is relegated to the appendix.

Lemma 1. Any lottery with mean $\mu$ and $S$ possible payoffs can be decomposed into a compound lottery of $S-1$ binary lotteries with mean $\mu$.

Let $x_{1}$ with $E x_{1}=\mu$ have $S=m+n$ possible outcomes in the union of $A^{-}=\left\{a_{1}^{-}, \ldots, a_{m}^{-}\right\}$ and $A^{+}=\left\{a_{1}^{+}, \ldots, a_{n}^{+}\right\}$, with $a_{1}^{-}<\ldots<a_{m}^{-}<\mu<a_{1}^{+}<\ldots<a_{n}^{+}$. Let us decompose this lottery into $x_{1} \sim\left(x^{1}, \pi^{1} ; \ldots ; x^{S-1}, \pi^{S-1}\right)$, where $x^{k} \sim\left(a^{k-}, p^{k}, a^{k+}, 1-p^{k}\right)$ is a $\mu$-mean binary lottery with $a^{k-} \in A^{-}, a^{k+} \in A^{+}$, and $p^{k} \in[0,1]$, for all $k \in\{1, S-1\}$. For each lottery $x^{k}$, define $y^{k}$ that takes value $\mu$ if $x^{k}=a^{k-}$, and $a^{k+}$ otherwise. It is then obvious that

$$
E U\left(x_{1}, y^{M}\right)-E U\left(\mu, y^{M}\right)=\sum_{k=1}^{S-1} \pi^{k}\left[E U\left(x^{k}, y^{k}\right)-E U\left(\mu, y^{k}\right)\right] .
$$

In other words, comparing risk contexts $\left(x_{1}, y^{M}\right)$ and $\left(\mu, y^{M}\right)$ can be performed by comparing $S-1$ pairs of risk contexts $\left(x^{k}, y^{k}\right)$ and $\left(\mu, y^{k}\right)$. Building on what we know on these comparisons from Section 4.1, we obtain the following results.

Proposition 3. Consider any menu $M$ containing two lotteries, a risky $x_{1}$ and a safe $x_{2}$ with the same mean. Let random variable $y^{M}$ denote the forgone best alternatives associated to $M$. The distribution of $\left(x_{1}, y^{M}\right)$ can be obtained from the distribution of $\left(x_{2}, y^{M}\right)$ through a sequence of mean-preserving spreads in $x \mid y^{M}$ and of marginal-preserving increases in concordance between $x$ and $y$.

Combining this with Proposition 2, this immediately implies the following corollary.

Corollary 2. Consider menu $M=\left\{x_{1}, x_{2}\right\}$, where $x_{2}$ takes value $E x_{1}=\mu$ with certainty. Risk-averse and regret-risk-seeking agents always prefer the safe choice in this menu. If the 
riskiness of $x_{1}$ is small in the sense of Arrow-Pratt, then the risky choice can potentially be preferred only if the local aversion to risk of regret is larger than half the local aversion to risk, i.e., iff $\rho(\mu, \mu) \geq 0.5 \alpha(\mu, \mu)$.

It is interesting to examine how a local mean-preserving spread in the distribution of the risky lottery $x_{1}$ in menu $M$ affects its attractiveness. As we will show, it is important to determine whether this MPS takes place to the regret domain or in the no-regret domain, where regret is defined from the point of view of the risk-taker. To examine this, let us formally decompose lottery $x_{1}$ into its regret and no-regret domains, where the regret domain corresponds to the state of nature in which the payoff of the risky lottery is less than the payoff of the safe bet: $x_{1} \sim\left(k x_{+}, p_{+} ; k x_{-}, p_{-}\right)$, with supp $x_{+} \subset\left[\mu,+\infty\left[\right.\right.$, supp $\left.\left.x_{-} \subset\right]-\infty, \mu\right]$, $p_{+}>0, p_{-}>0, p_{+}+p_{-}=1$, and $p_{+} E x_{+}+p_{-} E x_{-}=\mu$. The risky lottery is preferred iff

$$
p_{+} E\left[U\left(x_{+}, x_{+}\right)-U\left(\mu, x_{+}\right)\right]+p_{-} E\left[U\left(x_{-}, \mu\right)-U(\mu, \mu)\right] \geq 0 .
$$

We can rewrite this condition as follows:

$$
p_{+} E u_{+}\left(x_{+}\right)+p_{-} E u_{-}\left(x_{-}\right) \geq 0,
$$

where functions $u_{+}$and $u_{-}$are defined as

$$
u_{+}(x)=U(x, x)-U(\mu, x)
$$

and

$$
u_{-}(x)=U(x, \mu)-U(\mu, \mu) .
$$

This is a model of state-dependent expected utility. Obviously, the concavity of utility function $u_{-}$in the regret domain is the same as the concavity of $U(x, \mu)$ with respect to $x$. This means that the agent is risk-averse in the regret domain, in the sense that any local MPS in the regret domain reduces the attractiveness of the risky lottery. Its risk aversion in this domain is measured by $\alpha(x, \mu)$. But the analysis is very different in the no-regret domain. Indeed, the local risk aversion of $u_{+}$in this domain of payoffs is measured by

$$
-\frac{u_{+}^{\prime \prime}(x)}{u_{+}^{\prime}(x)}=-\frac{U_{x x}(x, x)+2 U_{x y}(x, x)+U_{y y}(x, x)-U_{y y}(\mu, x)}{U_{x}(x, x)+U_{y}(x, x)-U_{y}(\mu, x)} .
$$

The sign of this index of risk aversion is ambiguous. When $x$ is only marginally larger than $\mu$, this can be approximated by

$$
-\frac{u_{+}^{\prime \prime}(\mu)}{u_{+}^{\prime}(\mu)}=\alpha(\mu, \mu)-2 \rho(\mu, \mu) .
$$

These results are summarized in the following proposition.

Proposition 4. Consider menu $M=\left\{x_{1}, x_{2}\right\}$, where $x_{2}$ takes value $E x_{1}=\mu$ with certainty. Any mean-preserving spread of $x_{1}$ in the regret domain $x \leq \mu$ reduces the attractiveness of the risky lottery. A mean-preserving spread of $x_{1}$ in a small neighborhood above $\mu$ reduces its attractiveness if and only if $\rho(\mu, \mu) \leq 0.5 \alpha(\mu, \mu)$. 
An alternative intuition of this result can be obtained by examining the case of small risks. The following proposition, whose proof is skipped, generalizes Proposition 2.

Proposition 5. Consider a menu $M=\left\{x_{1}, x_{2}\right\}$ in which the risky lottery $x_{1}$ and the safe bet $x_{2}$ have the same mean $k \mu$. Let $x_{1} \sim\left(k x_{+}, p_{+} ; k x_{-}, p_{-}\right)$with $x_{-} \leq_{p} \mu \leq_{p} x_{+}$. In this menu, the increase in expected utility when switching from the safe lottery $x_{2}$ to the risky one $x_{1}$ equals

$\frac{E U\left(x_{1}, y^{M}\right)-E U\left(0, y^{M}\right)}{U_{x}(0,0)}=k^{2}\left[p_{+}\left(\rho_{0}-0.5 \alpha_{0}\right) E\left(x_{+}-\mu\right)^{2}-0.5 p_{-} \alpha_{0} E\left(x_{-}-\mu\right)^{2}\right]+O\left(k^{3}\right)$,

where $\rho_{0}=\rho(\mu, \mu)$ and $\alpha_{0}=\alpha(\mu, \mu)$.

The intuition of this result goes as follows. For small risk, the outcome-risk premium is proportional to $\operatorname{Var}\left(x_{1}\right)$, with

$$
\operatorname{Var}\left(x_{1}\right)=k^{2}\left[p_{+} E\left(x_{+}-\mu\right)^{2}+p_{-} E\left(x_{-}-\mu\right)^{2}\right] .
$$

As we know from Section 3, this outcome-risk premium should be compared to the regretrisk premium which is proportional to $-\Delta \operatorname{cov}\left(x, y^{M}\right)$. The increase in the covariance in $(x, y)$ when shifting risk context from the safe choice yielding $\left(0, y^{M}\right)$ to the risky one $\left(x_{1}, y^{M}\right)$ equals

$$
-\Delta \operatorname{cov}\left(x, y^{M}\right)=k^{2} p_{+} E\left(x_{+}-\mu\right)^{2} .
$$

The first term of the right-hand side of equality (23) is the difference between these two premia, weighted respectively by $0.5 \alpha_{0}$ and $\rho_{0}$.

Proposition 5 confirms our earlier findings. For example, in the small, a regret-sensitive agent will never choose the risky option if her degree of regret-risk aversion is smaller than half her absolute risk aversion. Indeed, in that case, the two terms in the right-hand side of equation (23) are negative. Moreover, the bias in favor of the risky choice is increasing in $p_{+} E\left(x_{+}-\mu\right)^{2} / p_{-} E\left(x_{-}-\mu\right)^{2}$, which is a measure of positive asymmetry in the distribution of $x_{1}$. This generalizes our findings on the positive skewness bias of regret-risk-averse DM when there are more than two possible outcomes in the risky choice.

\section{Reinterpretation in the binary RDU framework}

Our results in the case of binary lotteries presented in Section 4 provide an explanation for some of the standard risk-seeking observations made by Tversky and Kahneman (1992), among many others. First, people often prefer a small probability of winning a large prize over the expected value of that prospect. Second, people also often prefer a large probability of losing a substantial amount of money over the expected loss of that prospect. In decision theory, these two phenomena are called the "possibility effect". In both cases, the risky choice in the menu is positively skewed. This implies that people who contemplate the safe choice particularly fear the risk of regret when the higher payoff materializes. In the RDU 
framework, this is usually explained by the hypothesis that people tend to overweight the low probability of the best outcome. In this section, we translate our findings into this RDU terminology.

The standard method to elicit probability distortions in RDU consists in asking respondents to evaluate their certainty equivalent payoff $c$ for various binary lotteries $x_{1} \sim$ $\left(x_{+}, p_{+} ; x_{-}, p_{-}\right)$, with $x_{-}<x_{+}$and $p_{-}+p_{+}=1$. Suppose that $x_{-}$and $x_{+}$are fixed, and that respondents are asked to elicit their certainty equivalent for different values of $p$, as in Tversky and Kahneman (1992) for example. Eliciting a certainty equivalent associated to a lottery places the regret-sensitive respondent in a situation to recognize that she is indifferent between $x_{1}$ and $c$ when confronted with menu $\left\{x_{1}, c\right\}$. In our ARR model, this certainty equivalent payoff is defined as follows

$$
p_{+} U\left(x_{+}, x_{+}\right)+\left(1-p_{+}\right) U\left(x_{-}, c\right)=p_{+} U\left(c, x_{+}\right)+\left(1-p_{+}\right) U(c, c) .
$$

Suppose that the utility function $U$ is multiplicative as described in the previous section: $U(x, y)=u(x) v(y)$. The absolute aversion to risk of regret equals $\rho(x, y)=v^{\prime}(y) / v(y)$. Under this specification, equation (26) can be rewritten as

$$
w\left(p_{+}\right) u\left(x_{+}\right)+\left(1-w\left(p_{+}\right)\right) u\left(x_{-}\right)=u(c),
$$

with

$$
w\left(p_{+}\right)=\frac{p_{+} v\left(x_{+}\right)}{p_{+} v\left(x_{+}\right)+\left(1-p_{+}\right) v(c)} .
$$

Equation (27) is the standard formulation of the binary RDU model. In our model, for each value of $p_{+}$, the probability-distortion $w$ is jointly determined with the certainty equivalent payoff $c$ by solving system (27)-(28) with respect to these two unknowns. We can prove that there is an interior solution to this system, as stated in the following proposition, whose proof is releguated to the appendix.

Proposition 6. Suppose that $U(x, y)=u(x) v(y)$ and that $u$ is increasing. System (27)-(28) has an interior solution with a certainty equivalent $c$ in $\left[x_{-}, x_{+}\right]$. If $v$ is increasing, i.e., if the DM is regret-risk-averse, then

- the probability weighting $w\left(p_{+}\right)$is larger than $p_{+}$for all $p_{+} \in[0,1]$;

- the probability weighting function is concave in the neighborhood of $p_{+}=0$ and is convex in the neighborhood of $p_{+}=1$.

Moreover, $w^{\prime}(0)=v\left(x_{+}\right) / v\left(x_{-}\right) \geq 1$ and $w^{\prime}(1)=1$. These results are reversed if the $D M$ is regret-risk-seeking.

Consider an external observer who knows the agent's utility function $u$ and who tries to elicit the weighting function $w$ by observing a set of certainty equivalent payoffs associated to different binary lotteries with the same support $\left(x_{-}, x_{+}\right)$. For each possible probability $p_{+}$ of the large payoff, the observer deduces $w\left(p_{+}\right)$from the observation of $c$ by solving equation 

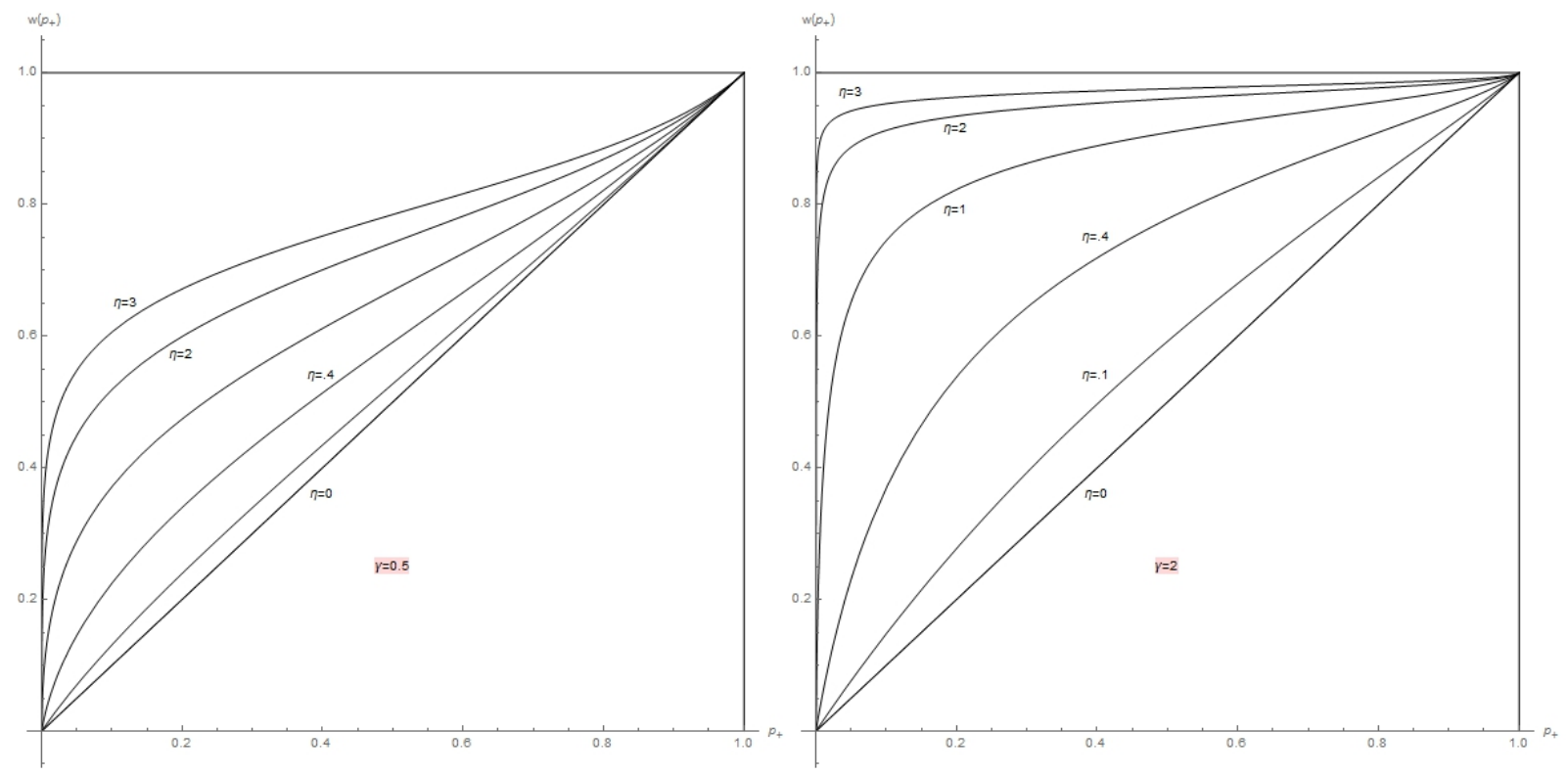

Figure 3: Probability transformation functions induced by the aversion to risk of regret. It is assumed that $x_{-}=1$ and $x_{+}=100, U(x, y)=x^{1-\gamma} y^{\eta} /(1-\gamma), \gamma$ is equal to 0.5 (left) or 2 (right), and $\eta \in\{0,0.1,0.4,1,2,3\}$.

(27). We showed in Proposition 6 that such an observer will deduce from this process that the agent is RDU with a probability weighting function $w$ that is optimistic $\left(w\left(p_{+}\right) \geq p_{+}\right)$if the agent is averse to risk of regret (and that is pessimistic if the agent is regret-risk-seeking). The effect of optimism can dominate the effect of risk aversion to induce the DM to prefer the risky choice, as shown in the previous section. Proposition 6 suggests that this probability weighting function is inverse-S shaped, since it is concave for low probabilities, and convex for large ones. In particular, this shows that ARR can explain the possibility effect, since $w^{\prime}(0)=v\left(x_{+}\right) / v\left(x_{-}\right) \geq 1$ : the ARR DM overweighs the probability of the best outcome. This overweighing is increasing in the degree of regret-risk aversion and in the distance between the two outcomes of the binary lottery.

However, as noticed earlier, this model is unable to generate both optimism for some probabilities and pessimism for others. Using the wording in Abdellaoui et al. (2010), our weighting function has an elevation which is too low. Moreover, because $w^{\prime}(1)=1$ in our framework, we are unable to obtain the "certainty effect" typically observed in experimental studies. ARR agents tend to underestimate the probability of unfavorable event and, at the limit of a very unlikely catastrophe, they tend to use the objective probability. We will come back to this question in Section 7.

To illustrate, we have drawn in Figure 3 the probability weighting function that would emerge from this elicitation process when considering lotteries with payoffs 1 and 100, and functions $u(x)=x^{1-\gamma} /(1-\gamma), \gamma \geq 0$, and $v(y)=y^{\eta}, \eta \geq 0$. Observe that $\rho(x, y)=\eta / y$, 
so that $\eta$ can be interpreted as an index of relative aversion to risk of regret. The left figure corresponds to a relatively low risk aversion of $\gamma=0.5$, whereas the right one corresponds to a larger risk aversion of $\gamma=2$. In each case, we have drawn the probability distortion functions associated to different degrees of relative regret-risk aversion $\eta=0,0.1,0.4,1,2$ and 3. Observe that an increase in ARR makes the agent more optimistic, with all probability weighting functions exhibiting a typical inverse-S shape. The high discrepancy between the true probability $p_{+}$and its distorted value $w\left(p_{+}\right)$when $p_{+}$is close to zero illustrates the possibility effect.

The two figures also illustrate the fact that the theory of regret aversion yields a probability weighting function that cannot be disentangled from the utility function $u$. They suggest in particular that more regret-risk-averse agents exhibit more optimism. It should also be emphasized that the probability weighting function $w$ is sensitive to the context given by $\left(x_{-}, x_{+}\right)$.

\section{The dual theory of elation-risk seeking}

Because only two-lottery menus were initially considered, the pioneering papers on regretrisk aversion by Loomes and Sugden (1982) and Bell (1982) allowed for both regret and rejoicing. Rejoicing (or elation) is the extra pleasure of knowing that, as matters turned out, one's choice generated a better outcome than the alternative. Quoting Loomes and Sugden (1982), elation is the extra pleasure of winning a prize as the consequence of one's own earlier risky decision rather than from an external action (an unanticipated tax rebate for example). By focusing the analysis on the forgone best alternative, Quiggin (1994) has oriented the literature towards regret, and this paper has been build on this foundation. This has been the price to pay to make regret aversion models useful for examining menus with more than two choices. But it is straightforward from our work to imagine a dual theory of elation-risk and of elation-risk aversion (or seeking). This is the objective of this section.

Consider again a decision-maker who is confronted to a menu $M$ of lotteries, as described in Section 2. Let $z^{M}(s)$ denote the forgone worst alternative of menu $M$ in state $s: z^{M}(s)=$ $\min _{x_{\theta} \in M} x_{\theta}(s)$. We say than the decision-maker is sensitive to rejoicing if her utility ex-post is a bivariate function of the actual consumption $x$ and of the forgone worst alternative $z^{M}$. The intensity $e$ of elation is measured by the difference between the actual payoff and the forgone worst alternative: $e(s)=x_{\theta}(s)-z^{M}(s)$. The ex-ante problem of a rejoicing-sensitive agent facing a menu $M$ is to select the lottery $x_{\theta}$ in $M$ that maximizes $E U\left(x_{\theta}, z^{M}\right)$.

As in Section 2, let us compare two risk contexts characterized respectively by $\left(x_{1}, z_{1}\right)$ and $\left(x_{2}, z_{2}\right)$. Let $F_{i}: \mathbb{R}^{2} \rightarrow \mathbb{R}$ denote the bivariate distribution function associated to context $i, i=1,2$. Suppose that $F_{2}$ is obtained from $F_{1}$ through a sequence of Marginal-Preserving Reductions in Concordance. By definition of MPRCs, this shift in the joint distribution of $(x, z)$ necessarily generates a Rothschild-Stiglitz increase in the risk of elation $e$. Elationrisk seekers are by definition people who like any such shift in the distribution of $(x, z)$, whereas elation-risk-averse people dislike any such shift. Obviously, elation-risk seekers have a bivariate utility function $U(x, z)$ that is submodular, whereas elation-risk-averse people 


\begin{tabular}{lcc}
\hline Ball number & $0-4$ & $5-99$ \\
\hline Lottery $x_{1}^{*}$ & 0 & 100 \\
Lottery $x_{2}$ & 95 & 95 \\
\hline$z^{12}$ & 0 & 95 \\
\hline Elation & 0 & 5 \\
\hline
\end{tabular}

\begin{tabular}{lcc}
\hline & $0-4$ & $5-99$ \\
\hline Lottery $x_{1}$ & 0 & 100 \\
Lottery $x_{2}^{*}$ & 95 & 95 \\
\hline$z^{12}$ & 0 & 95 \\
\hline Elation & 95 & 0 \\
\hline
\end{tabular}

Table 4: Elation-risk contexts in a menu $M_{12}$ with an urn containing 100 balls numbered from 0 to 99 .

have a supermodular $U(x, z)$.

Tversky and Kahneman (1992) have observed that the median certainty equivalent of lottery $x_{1} \sim(100,95 \% ; 0,5 \%)$ is 78 , which suggests an unrealistically large degree of risk aversion. They referred to the "certainty effect" to explain this puzzle. This cannot be explained by regret-risk aversion which tends to induce risk-seeking for binary lotteries, as shown in the previous section. A plausible explanation for the certainty effect is that people are elation-risk-seeking. To see this, consider Table 4 that describes a menu $M_{12}$ of two lotteries based on drawing a ball from an urn containing 100 balls numbered from 0 to 99 . Lottery $x_{1}$ is the one proposed by Tversky and Kahneman (1992) with a probability of $95 \%$ of winning 100. The alternative choice is to receive the expected gain 95 with probability 1 . It is clear that the safe choice yields a much riskier elation of 95 with probability of $5 \%$ than the risky choice. People who are elation-risk seekers would be particularly prone to the safe choice, and they will require a particularly large premium to accept this highly negatively skewed lottery. To sum up, the certainty effect suggests that people are elation-risk seekers.

As in Section 4, let us examine a menu that contains two lotteries, a safe lottery yielding a zero payoff, and a risky lottery $x_{1}$ yielding either $-k$ with probability $1-p$ or $K=k(1-p) / p$ with probability $p$, with $k>0$ and $p \in[0,1]$. In Figure 4 , we draw the transfers of probability masses in the $(x, z)$-space that is necessary to go from the safe choice to the risky choice. As illustrated in this figure, it can be shown that it is always true that one can go from the safe to the risky choice by a sequence of two conditional mean-preserving spreads in actual payoff and one marginal-preserving increase in concordance between $x$ and $z$. This MPIC implies a reduction in the elation-risk. In other words, the safe choice always entails an increase in the risk of elation, something that elation-risk seekers like.

It is easy to check that switching from the safe to the risky asset in this menu raises expected utility by

$$
E U\left(x_{1}, z^{M}\right)-E U\left(0, z^{M}\right)=\left[p \psi_{0}-0.5 \alpha_{0}\right] U_{x}(0,0) \operatorname{Var}\left(x_{1}\right)+O\left(k^{3}\right),
$$

where $\psi(a, b)=U_{x z}(a, b) / U_{x}(a, b)$ is the index of absolute elation-risk aversion and $\psi_{0}=$ $\psi(0,0)$. We see that elation-risk seeking goes with risk aversion to reject the risky lottery. We also see from this equation that the effect of elation-risk seeking is larger when the probability of success is larger, i.e., when the risky choice yields a negatively skewed payoff. 


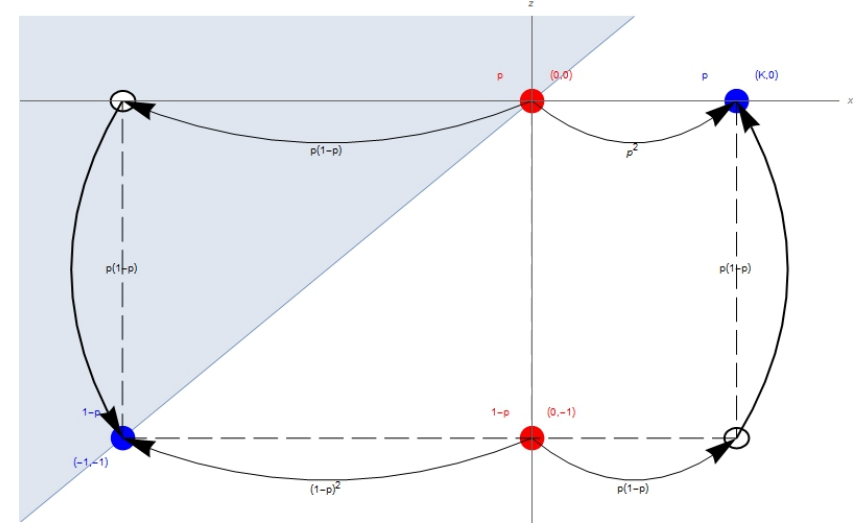

Figure 4: Transfers of probability masses in the $(x, z)$-space in the context of a menu $M=$ $\left\{x_{1} \sim(K, p ;-1,1-p), x_{2}={ }_{p} 0\right\}$.

Following the method developed in Section 5, it is straightforward to extend these findings to a one-risky-one-safe menu in which the risky choice has more than two outcomes.

We can also link elation-risk seeking to the RDU distortion of probabilities. Consider again the problem of determining the certainty equivalent of a lottery $x_{1} \sim\left(x_{+}, p_{+} ; x_{-}, p_{-}\right)$. As in Section 4.3, let us interpret this certainty equivalent $c$ as the payoff of a safe lottery $x_{2}$ that makes the agent indifferent between the two lotteries in menu $M_{12}$. If we assume that $U(x, z)=u(x) h(z)$, it is therefore defined by the following condition:

$$
w\left(p_{+}\right) u\left(x_{+}\right)+\left(1-w\left(p_{+}\right)\right) u\left(x_{-}\right)=u(c),
$$

where function $w$ is the probability-distortion function defined as follows:

$$
w\left(p_{+}\right)=\frac{p_{+} h(c)}{p_{+} h(c)+\left(1-p_{+}\right) h\left(x_{-}\right)} .
$$

Equations (30) and(31) is a system of two equations with two unknowns, $c$ and $w$. It can be shown that function $w$ has the following characteristics. First, $w^{\prime}(0)=1$, which confirms that the elation-risk attitude does not affect the attitude towards highly skewed lotteries. In other words, elation-risk seeking cannot explain the possibility effect. Moreover, under elation-risk seeking, the agent is pessimistic $(w(p) \leq p)$, and $w$ is locally concave and convex in the neighborhood of 0 and 1 respectively. These properties are reversed if the agent is elation-risk-averse. Figure 5 illustrates these findings.

When comparing this figure with Figure 3, a clear picture emerges from these two theories on regret and elation. Regret-risk aversion can explain the "possibility effect" associated to lotteries with a low probability of the best payoff (large $w^{\prime}(0)$ ). Elation-risk seeking can explain the "certainty effect" associated to lotteries with a low probability of the worst payoff (large $w^{\prime}(1)$ ). It is therefore tempting to associate the two concepts in the same preference functional. We do this in an informal way in this paper, paving the way for further research 


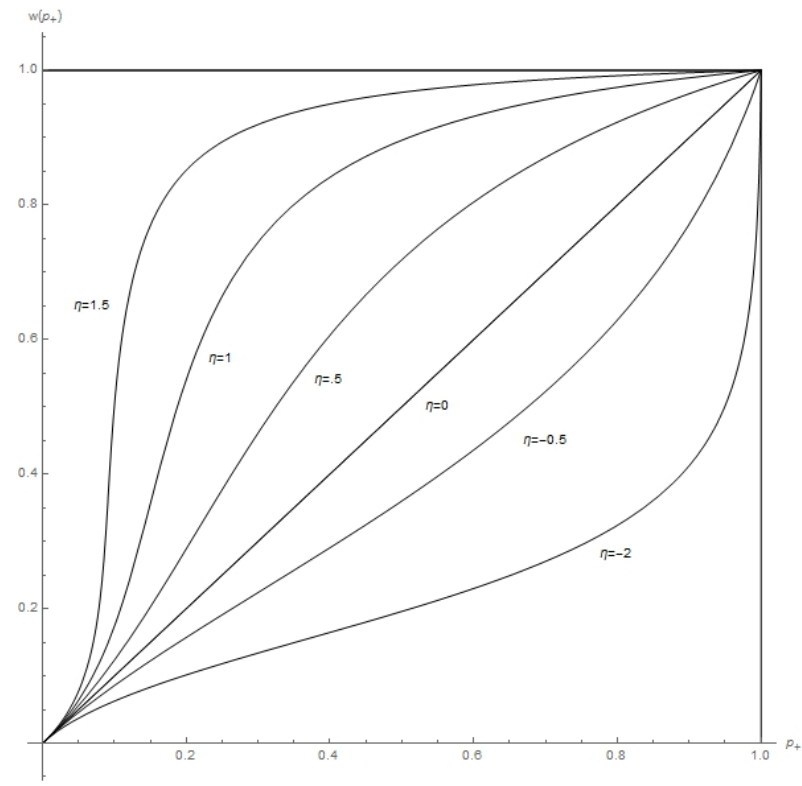

Figure 5: Probability transformation function induced by the sensitivity to elation-risk. It is assumed that $x_{-}=1$ and $x_{+}=10, U(x, z)=x^{1-\gamma} z^{\eta} /(1-\gamma)$. Relative risk aversion $\gamma$ is equal to 0.5 , and the absolute aversion to elation-risk is $\eta \in\{-2,-0.5,0,0.5,1,1.5\}$.

in this domain. In order to model preferences that combine the anticipation of regret and elation, let us consider a utility function $U$ with three variables: the actual consumption $x(s)$, the forgone best alternative $y^{M}(s)=\max _{x_{\theta} \in M} x_{\theta}(s)$, and the forgone worst alternative $z^{M}(s)=\min _{x_{\theta} \in M} x_{\theta}(s)$. Suppose that

$$
U(x, y, z)=u(x) v(y) h(z)
$$

with $u(x)=x^{1-\gamma} /(1-\gamma), v(y)=y^{\eta_{y}}$ and $h(z)=z^{\eta_{z}}$, where $\gamma$ is relative risk aversion, $\eta_{y}=\rho / y$ is relative regret-risk aversion, and $\eta_{z}=\psi / z$ is relative elation-risk aversion. In a menu containing a risky lottery $\left(x_{+}, p_{+} ; x_{-}, p_{-}\right)$and its certainty equivalent $c$, the certainty equivalent must satisfy condition (30) with

$$
w\left(p_{+}\right)=\frac{p v\left(x_{+}\right) h(c)}{p v\left(x_{+}\right) h(c)+(1-p) v(c) h\left(x_{-}\right)} .
$$

This probability weighting function is described in Figure 6 when $x_{-}=1, x_{+}=2, \gamma=0.5$, $\eta_{y}=2.3$, and $\eta_{z}=3.5$. We also draw in this figure the inverse-S weighting function that was suggested by Tversky and Kahneman (1992):

$$
w(p)=\frac{p^{h}}{\left(p^{h}+(1-p)^{h}\right)^{1 / h}},
$$

with $h=0.61$. The almost perfect fit between these two curves suggests that the probability distortions appearing in the RDU literature could be made endogenous by combining expected utility with regret-risk aversion and elation-risk seeking. 


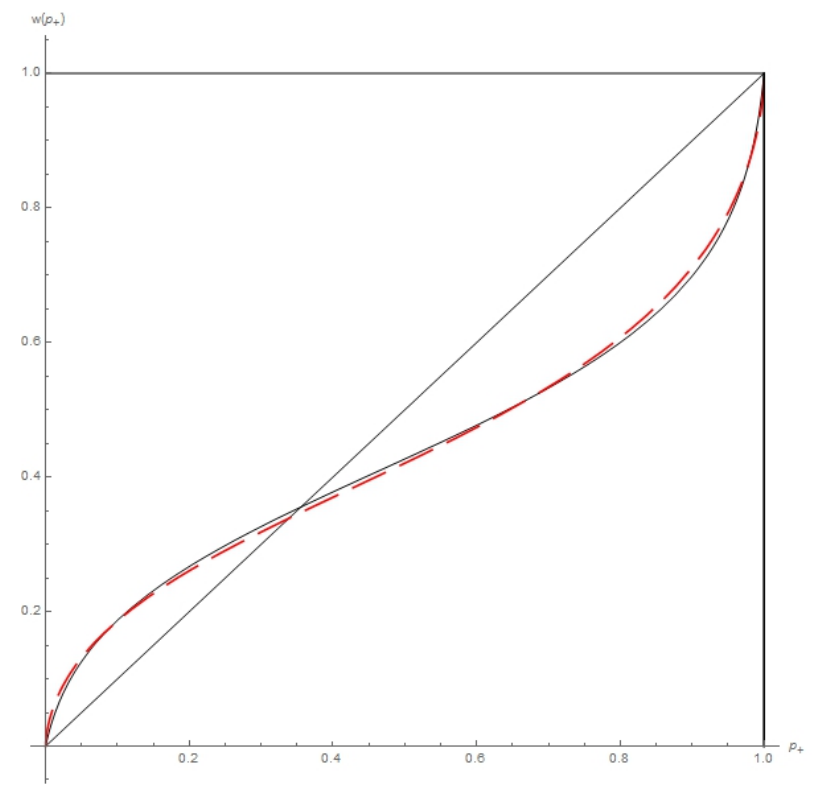

Figure 6: Probability distortion function (plain curve) induced by the sensitivity to both regret-risk and elation-risk. It is assumed that $x_{-}=1$ and $x_{+}=2, U(x, y, z)=$ $x^{1-\gamma} y^{\eta_{y}} z^{\eta_{z}} /(1-\gamma)$. We assume that $\gamma=0.5, \eta_{y}=2.3$ and $\eta_{z}=-3.5$. The dashed curve corresponds to the weighting function (34) with $h=0.61$, as proposed by Tversky and Kahneman (1992). 


\section{The portfolio problem with regret and rejoicing}

In this section, we apply our definition of regret-risk aversion and elation-risk seeking to the static one-risky-one-safe-asset portfolio. Consider an investor with initial wealth $z$ who can invest in a safe asset whose return is normalized to zero and in a risky asset whose return is a random variable $x$ with a known distribution function $F$. We normalize $z$ to unity. To make the problem interesting, let us suppose that 0 is in the interior of the support of $x$. If $k$ denotes the share of wealth invested in the risky asset, final wealth is $1+k x$. Let us assume that the equity share $k$ is restricted to belong to $[\underline{k}, \bar{k}]$ for some arbitrary pair $(\underline{k}, \bar{k}) \in \mathbb{R}^{2}$ such that $\underline{k}<\bar{k}$. A typical example is $\underline{k}=0$ in which shorting the risky asset is prohibited. Another example is $\bar{k}=1$, in which borrowing at the risk-free rate to invest in the risky asset is prohibited. This analysis generalizes what has been done before in this paper by allowing more than two choices in the menu. ${ }^{14}$

The portfolio menu is $M=\{1+k x \mid k \in[\underline{k}, \bar{k}]\}$. The forgone best and worst alternatives associated to this menu are as follows:

$$
y^{M}(s)=\left\{\begin{array}{l}
1+\underline{k} x \text { if } x \leq 0 \\
1+\bar{k} x \text { if } x>0,
\end{array}\right.
$$

and

$$
z^{M}(s)=\left\{\begin{array}{l}
1+\bar{k} x \text { if } x \leq 0 \\
1+\underline{k} x \text { if } x>0 .
\end{array}\right.
$$

The decision problem can be written as

$$
k^{*} \in \arg \max _{k \in[\underline{k}, \bar{k}]} V(k ; \underline{k}, \bar{k})=E U\left(1+k x, y^{M}, z^{M}\right) .
$$

Notice that the objective function $V$ is concave in $k$, so that the first-order condition is necessary and sufficient. We hereafter suppose that the solution is interior, so that this condition is

$$
\frac{\partial V}{\partial k}\left(k^{*} ; \underline{k}, \bar{k}\right)=\operatorname{Ex}_{x}\left(1+k^{*} x, y^{M}, z^{M}\right)=0 .
$$

We assume that $k^{*}$ is positive. With an interior solution, the agent always feels a mixture of regret and elation. For example, when the excess return is positive, the agent experiences regret from not having invested the maximum $\bar{k}$ in the risky asset, and at the same time she is elated from having invested something in the risky asset. We are interested in determining the impact of a change in the lower and upper constraints on the optimal portfolio allocation $k^{*}{ }^{15}$

\footnotetext{
${ }^{14}$ There exist other interpretations of this model. For example, consider the case of an insurable risk of loss $\ell$, which is random. A coinsurance contract can be purchased in which the policyholder with initial wealth $z_{0}$ gets indemnity $(1-k) \ell$ ex post against the payment of a premium $(1-k) P$ ex ante, where $k$ is the retention rate, and $P$ is the full insurance premium. Final wealth is thus $z_{0}-\ell+(1-k) \ell-(1-k) P$, which can be rewritten as $z+k x$, with $z=z_{0}-P$ and $x=P-\ell$. Sandmo (1971) provides another application for the case of a price-taking firm that has to determine its capacity before knowing the demand.

${ }^{15}$ The welfare analysis is immediate in the case of regret. As shown by Sarver (2008), if $U_{y}<0$, i.e., if the investor is averse to regret, any reduction in the choice set (increase in $\underline{k}$, reduction in $\bar{k}$ ) that does not eliminate the optimal solution $k^{*}(\underline{k}, \bar{k})$ raises welfare ex ante. See also Huang et al. (2014).
} 
Because $V$ is concave in $k$, this comparative static analysis is driven by the cross-derivatives of $V$. We have that

$$
\left.\frac{\partial^{2} V}{\partial k \partial \underline{k}}\right|_{k=k^{*}}=\int_{-\infty}^{0} x^{2} U_{x y}\left(1+k^{*} x, y^{M}, z^{M}\right) d F(x)+\int_{0}^{-\infty} x^{2} U_{x z}\left(1+k^{*} x, y^{M}, z^{M}\right) d F(x) .
$$

This is unambiguously positive under the joint assumptions of regret-risk aversion and elationrisk aversion. Under these conditions, increasing the minimum risk exposure $\underline{k}$ always raises the optimal risk exposure $k^{*}$. Similarly, we have that

$$
\left.\frac{\partial^{2} V}{\partial k \partial \bar{k}}\right|_{k=k^{*}}=\int_{-\infty}^{0} x^{2} U_{x z}\left(1+k^{*} x, y^{M}, z^{M}\right) d F(x)+\int_{0}^{+\infty} x^{2} U_{x y}\left(1+k^{*} x, y^{M}, z^{M}\right) d F(x) .
$$

This is also positive under regret-risk aversion and elation-risk aversion. This yields the following proposition.

Proposition 7. Consider the portfolio problem in which final wealth is $1+k x$, with $k \in$ $[\underline{k}, \bar{k}]$, and suppose that the optimal solution $k^{*}$ is interior. Raising the lower limit $\underline{k}$ or the upper limit $\bar{k}$ of the risk exposure always raises the optimal risk exposure $k^{*}$ under the joint assumptions of regret-risk aversion and elation-risk aversion.

This means that regret-risk aversion and elation-risk aversion tend to push the optimal risk exposure to the center of the opportunity set. An extreme illustration of this phenomenon is obtained when assuming that the equity premium is zero, so that $E x=0$. As is wellknown, in the absence of counterfactual emotion, the optimal portfolio is fully invested in the safe asset in that case. But it is a simple extension of the above proposition that imposing a no-borrowing constraint $\underline{k}=0$ will induce the regret-risk-averse investor to accept some equity risk in her portfolio. Braun and Muermann (2004), Muermann et al. (2006) and Michenaud and Solnik (2008) derived this result respectively in the context of insurance, portfolio choice and currency hedging decisions, under the Bell's specification $U(x, y)=$ $u(x)-R(u(y)-u(x)) .{ }^{16}$ Our result generalizes these findings to any preferences that exhibits regret-risk aversion and/or elation-risk aversion.

Of course, these results are reversed when the agent is regret-risk seeking and/or elationrisk seeking. The comparative statics analysis is more complex in the case of regret-risk aversion combined with elation-risk seeking. Observe also that one can rewrite the first-order condition (38) by using an indirect utility function $u$ :

$$
\operatorname{Exu}^{\prime}\left(1+k^{*} x\right)=0
$$

with

\footnotetext{
${ }^{16}$ These results illustrate again the fact that the optimal choices of regret-sensitive DMs are menu-dependent. Expanding the number of options in the menu changes the nature of the choice problem. In Gollier and Salanié (2012), we explore the portfolio choice problem when the number of assets is large enough to make markets complete.
} 


$$
u^{\prime}(w)=\left\{\begin{array}{lll}
U_{x}\left(W_{-}(w)\right) & \text { if } \quad w<1 \\
U_{x}\left(W_{+}(w)\right) & \text { if } \quad w \geq 1
\end{array}\right.
$$

where $W_{-}(w)=(w, 1+\underline{m}(w-1), 1+\bar{m}(w-1)), W_{+}(w)=(w, 1+\bar{m}(w-1), 1+\underline{m}(w-1))$, $\underline{m}=\underline{k} / k^{*}$, and $\bar{m}=\bar{k} / k^{*}$. The index of absolute risk aversion of this indirect utility function equals

$$
-\frac{u^{\prime \prime}(w)}{u^{\prime}(w)}= \begin{cases}\alpha\left(W_{-}(w)\right)-\underline{m} \rho\left(W_{-}(w)\right)-\bar{m} \psi\left(W_{-}(w)\right) & \text { if } \quad w<1 \\ \alpha\left(W_{+}(w)\right)-\bar{m} \rho\left(W_{+}(w)\right)-\underline{m} \psi\left(W_{+}(w)\right) & \text { if } \quad w>1\end{cases}
$$

where $\alpha=-U_{x x} / U_{x}$ is risk aversion, $\rho=U_{x y} / U_{x}$ is regret-risk aversion and $\psi=U_{x z} / U_{x}$ is elation-risk aversion. Suppose that these indices are approximately constant in the relevant support of $W_{-}(w)$ and $W_{+}(w)$. The above equation then shows that regret-risk aversion plays a more important role to reduce risk aversion in the gain domain $(w>1)$ than in the loss domain $(w<1)$. Similarly, elation-risk seeking plays a more important role to raise risk aversion in the loss domain than in the gain domain. To keep the analysis simple, suppose that $\underline{k}=\underline{m}=0$. Then, the index of risk aversion of $u$ goes down from $\alpha(\mathbf{1})-\bar{m} \psi(\mathbf{1})>\alpha(\mathbf{1})$ for small negative excess returns to $\alpha(\mathbf{1})-\bar{m} \rho(\mathbf{1})<\alpha(\mathbf{1})$ for small positive excess returns, where 1 is the triplet of 1 . This suggests that the impact of a mean-preserving spread in returns reduces the demand for the risky asset less if it concentrated in the domain of positive excess returns than in the domain of negative excess returns. If counterfactual emotions are large enough compared to risk aversion, such MPS in the gain domain can even raises the demand for the risky asset. ${ }^{17}$ This is another illustration of the longshot bias that is generated by regret-risk aversion.

\section{Conclusion}

In spite of its intuitive appeal and the many testable predictions of the theory, regret-risk aversion has received relatively little attention by economists. A possible explanation is the relatively weak theoretical foundation of our regret model. The theory has no clear definition of what actually is regret and regret aversion, or regret-risk and regret-risk aversion. For the sake of comparison, the axiomatic development of the EU theory after WWII has quickly been followed by the building of the crucial tools of an index of risk aversion and of stochastic dominance orders. This has opened the door to a myriad of applications in finance, macroeconomics and IO researches for example. No such evolution has been possible in regret theory. In this paper, we tried to fill this gap by proposing a coherent theory and measurement of regret risk and regret risk aversion. We did that by using a general formulation in which the decision maker maximizes the expectation of a bivariate utility function which is not only sensitive to the actual payoff of the chosen act, but also to the best alternative payoff if another action would have been selected ex ante.

When the decision maker is confronted to a menu of lotteries, we have defined regret in each state as the difference between the best possible payoff and the actual payoff associated

\footnotetext{
${ }^{17}$ As shown by equation (41) and discussed in Rothschild and Stiglitz (1971) and Gollier (1995), what matters to determine the impact of a MPS in the distribution of returns on the asset demand is the concavity of the function $x u^{\prime}\left(1+k^{*} x\right)$.
} 
to the chosen lottery. We used the concept of comparative concordance, which is a stochastic order useful to measure the degree of dependence between two random variables. We have shown that a reduction in concordance between the forgone best alternative and the actual payoff yields an increase in risk of regret, without affecting the marginal distributions of these two random variables. It is thus natural to define the notion of regret-risk aversion by requiring that ex-ante welfare is reduced by any such reduction in concordance. We have shown that this requires the bivariate utility function to be supermodular. We have defined accordingly the concept of regret-risk premium together with a local index of regret-risk aversion, and we have shown that in the small, the former is proportional to the latter. More importantly, we have shown that regret-risk-averse agents exhibit a natural bias in favor of positively skewed risks. If the menu contains a risky lottery and its certainty equivalent, as is the case in most experiments used to elicit preferences under risk, the behavior of regret-riskaverse agents is equivalent to the one of rank-dependent utility agents model which would use a probability distortion function that exhibits both optimism and an inverse-S shape. But if regret-risk aversion can explain the possibility effect, it cannot explain the certainty effect. The dual theory of elation-risk seeking can do that. We have suggested that combining regret-risk aversion and elation-risk seeking is able to explain the two effects, and that it can duplicate the inverse-S probability weighting functions that have been estimated in the literature. 


\section{Appendix 1: Proof of Proposition 6}

Eliminating $w$ from (27)-(28) yields

$$
F(c)=\frac{p_{+} v\left(x_{+}\right)}{\left(1-p_{+}\right) v(c)+p_{+} v\left(x_{+}\right)} u\left(x_{+}\right)+\frac{\left(1-p_{+}\right) v(c)}{\left(1-p_{+}\right) v(c)+p_{+} v\left(x_{+}\right)} u\left(x_{-}\right)-u(c)=0
$$

We have that

$$
F\left(x_{-}\right)=\frac{p_{+} v\left(x_{+}\right)}{\left(1-p_{+}\right) v\left(x_{-}\right)+p_{+} v\left(x_{+}\right)}\left(u\left(x_{+}\right)-u\left(x_{-}\right)\right) \geq 0
$$

and

$$
F\left(x_{+}\right)=-\left(1-p_{+}\right)\left(u\left(x_{-}\right)-u\left(x_{+}\right)\right) \leq 0 .
$$

Because $F$ is continuous, there must exists a real $c \in\left[x_{-}, x_{+}\right]$such that $F(c)=0$. This must be the solution of system (27)-(28). Because $c$ is less than $x_{+}$, its is immediate from (28) that $w\left(p_{+}\right)$is larger than $p_{+}$if $v$ is increasing.

We now examine the shape of the probability weighting function. To do this, we fully differentiate system (27)-(28) with respect to $p_{-}$. It yields (we simplify the notation by replacing $p_{+}=p$ )

$$
\frac{d c}{d p}=\frac{u\left(x_{+}\right)-u\left(x_{-}\right)}{u^{\prime}(c)} \frac{d w}{d p}
$$

and

$$
\frac{d w}{d p}=\frac{w v(c)+(1-w) v\left(x_{+}\right)}{(1-p) v(c)+p v\left(x_{+}\right)+(1-p) w v^{\prime}(c) \frac{u\left(x_{+}\right)-u\left(x_{-}\right)}{u^{\prime}(c)}} .
$$

Let us first examine the case $p=1$. The above equations imply that $c=x_{+}$and $w=1$, and $w^{\prime}(1)=1$. Moreover differentiating the above equation around $p=1$ yields

$$
\left.\frac{d^{2} w}{d p^{2}}\right|_{p=1}=\frac{2 v^{\prime}\left(x_{+}\right)\left(u\left(x_{+}\right)-u\left(x_{-}\right)\right)}{u^{\prime}\left(x_{+}\right) v\left(x_{+}\right)} \geq 0
$$

Let us alternatively consider the case $p=0$, which implies that $c=x_{-}, w=0, c^{\prime}(0)=$ $\left(u\left(x_{-}\right)-u\left(x_{+}\right) v\left(x_{+}\right) / u^{\prime}\left(x_{-}\right) v\left(x_{-}\right)\right.$and $w^{\prime}(0)=v\left(x_{+}\right) / v\left(x_{-}\right)$. Finally, differentiating equation (48) in the neighborhood of $p=0$ yields

$$
\left.\frac{d^{2} w}{d p^{2}}\right|_{p=0}=-\frac{2 v\left(x_{+}\right)^{2} v^{\prime}\left(x_{-}\right)\left(u\left(x_{+}\right)-u\left(x_{-}\right)\right)}{u^{\prime}\left(x_{-}\right) v\left(x_{-}\right)^{3}}-\frac{2 v\left(x_{+}\right)\left(v\left(x_{+}\right)-v\left(x_{-}\right)\right)}{v\left(x_{-}\right)^{2}} \leq 0 .
$$

This concludes the proof of Proposition 6 .

\section{Appendix 2: Proof of Lemma 1}


Without loss of generality, let $\mu$ be zero. Let $S=m+n$, and let the risky lottery $x_{1}$ in menu $M$ be

$$
x_{1}=x_{1}^{0} \sim\left(a_{1}^{-}, p_{1}^{-} ; \ldots ; a_{m}^{-}, p_{m}^{-} ; a_{1}^{+}, p_{1}^{+} ; \ldots ; a_{n}^{+}, p_{n}^{+}\right),
$$

with $a_{1}^{-}<\ldots<a_{m}^{-}<0<a_{1}^{+}<\ldots<a_{n}^{+}, p_{i}^{-}>0, i=1, \ldots, m, p_{j}^{+}>0, j=1, \ldots, n$, and $\sum_{i=1}^{m} p_{i}^{-}+\sum_{j=1}^{n} p_{j}^{+}=1$. Assume that $E x_{1}=0$. Define $p_{i j}$ as

$$
p_{i j}=\frac{-a_{i}^{-}}{a_{j}^{+}-a_{i}^{-}} \in[0,1]
$$

Define lottery $x_{i j} \sim\left(a_{j}^{+}, p_{i j} ; a_{i}^{-}, 1-p_{i j}\right)$. By construction, $E x_{i j}=0$. Initialize the probability vector $Q=\left(q_{1}^{0-}, \ldots, q_{m}^{0-}, q_{1}^{0+}, \ldots, q_{n} 0+\right)$ such that for all $i, q_{i}^{0,-}=p_{i}^{-}$and for all $j, q_{j}^{0+}=p_{j}^{+}$. We also initialize two sets $I=J=\{\emptyset\}$.

We consider the following $n+m-2$ iterations. At the beginning of iteration $k, I \cup J$ contains the $k-1$ states of nature whose lottery's initial payoff has been replaced by a binary zero-mean lottery.

Iteration k: Take an arbitrary pair $(i, j), i \in\{1, \ldots, m\} / I, j \in\{1, \ldots, n\} / J$. Consider two cases.

Case 1: Suppose that $q_{i}^{k-1-}<-q_{j}^{k-1+} a_{j}^{+} / a_{i}^{-}$. Then, define $\pi^{k}=q_{i}^{k-1-}\left(a_{j}^{+}-a_{i}^{-}\right) / a_{j}^{+}$. Perform the following two operations on lottery $x^{k-1}$ :

- Replace the atom $a_{i}^{-}$by lottery $x_{i j}$, and raises the associated probability $q_{i}^{k-1-}$ up to $q_{i}^{k-}=\pi^{k}$.

- Reduce the probability associated to $a_{j}^{+}$from $q_{j}^{k-1+}$ to $q_{j}^{k+}=q_{j}^{k-1+}+\left(q_{i}^{k-1-} a_{i}^{-} / a_{j}^{+}\right)>$ 0 .

Moreover, append state $i$ into the set of negative states whose initial payoff $a_{i}^{-}$as been replaced by a binary zero-mean lottery $x_{i j}: I^{k}=I^{k-1} \cup i$.

Case 2: Suppose that $q_{i}^{k-1-} \geq-q_{j}^{k-1+} a_{j}^{+} / a_{i}^{-}$. Then, define $\pi^{k}=-q_{j}^{k-1+}\left(a_{j}^{+}-a_{i}^{-}\right) / a_{i}^{-}$. Perform the following two operations on lottery $x^{k-1}$ :

- Replace the atom $a_{j}^{+}$by lottery $x_{i j}$, and raises the associated probability $q_{j}^{k-1+}$ up to $q_{j}^{k+}=\pi^{k}$.

- Reduce the probability associated to $a_{i}^{-}$from $q_{i}^{k-1-}$ to $q_{i}^{k-}=q_{i}^{k-1-}+\left(q_{j}^{k-1+} a_{j}^{+} / a_{i}^{-}\right) \geq$ 0 .

Moreover, append state $j$ into the set of positive states whose initial payoff $a_{j}^{+}$as been replaced by a binary zero-mean lottery $x_{i j}: J^{k}=J^{k-1} \cup j$.

In both cases, this procedure yields a new lottery $x^{k}$ that has the same distribution of payoffs, but in which one payoff has been replaced by a binary zero-mean lottery. After 
$m+n-2$ iterations, all payoffs have replaced by such lotteries, expect two of them. By construction, since the $E x^{k}=0$, the remaining two atoms $\left(a_{i}^{-}, a_{j}^{+}\right)$must be opposite in sign and have remaining probabilities $q_{i}^{m+n-2-}$ and $q_{j}^{m+n-2+}$ such that

$$
a_{i}^{-} q_{i}^{m+n-2-}+a_{j}^{+} q_{j}^{m+n-2+}=0 .
$$

This concludes the proof of Lemma 1.

\section{References}

Abdellaoui, M. (2000): "Parameter-Free Elicitation of Utility and Probability Weighting Functions," Management Science, 46, 1497-1512.

Abdellaoui, M., O. L'Haridon, and H. Zank (2010): "Separating curvature and elevation: A parametric probability weighting function," Journal of Risk and Uncertainty, 41, $39-65$.

Ali, M. M. (1977): "Probability and utility estimates for racetrack bettors," Journal of Political Economy, 85, 803-815.

Atkinson, A. And F. Bourguignon (1982): "The comparison of multi-dimensioned distributions of economic status," Review of Economic Studies, 49, 183-201.

BARRo, R. (2006): "Rare disasters and asset markets in the twentieth century," Quarterly Journal of Economics, 121, 823-866.

- (2009): "Rare disasters, asset prices, and welfare costs," American Economic Review, 99, 243-264.

BELL, D. E. (1982): "Regret in decision making under uncertainty," Operations Research, 30, 961-981.

(1983): "Risk premiums for decision regret," Management Science, 29, 1156-1166.

Bhattacharya, N. And T. Garrett (2008): "Why people choose negative expected return assets - an empirical examination of a utility theoretic explanation," Applied Economics, $40,27-34$.

Bikhchandani, S. And U. Segal (2011): "Transitive regret," Theoretical Economics, 6, 95-108.

Bleichrodt, H. And P. Wakker (2015): "Regret Theory: A Bold Alternative to the Alternatives," Economic Journal, 125, 493-532.

Braun, M. And A. Muermann (2004): "The impact of regret on the demand for insurance," Journal of Risk and Insurance, 71, 737-767.

Brehaut, J. C., A. O'Connor, T. Wood, T. Hack, L. Siminoff, E. Gordon, and D. Feldman-Stewart (2003): "Validation of a decision regret scale," Medical Decision Making, 23, 281-292. 
Camille, N., G. Coricelli, J. Sallet, P. Pradat-Diehl, J. Duhamel, and A. Sirigu (2004): "The Involvement of the Orbitofrontal Cortex in the Experience of Regret," Science, 304, 1167-1170.

Chapman, G. And E. Coups (2006): "Emotions and preventive health behavior: Worry, regret, and influenza vaccination," Health Psychology, 25, 82-90.

Eeckhoudt, L., B. Rey, And H. Schlesinger (2007): "A good sign for multivariate risk taking," Management Science, 53, 117-124.

Eeckhoudt, L. And H. Schlesinger (2006): "Putting risk in its proper place," American Economic Review, 96, 280-289.

EichneR, T. And A. WAgener (2011): "Increases in skewness and three-moment preferences," Mathematical Social Sciences, 61, 109-113.

Epstein, L. And S. TANny (1980): "Increasing generalized correlation: A definition and some economic consequences," Canadian Journal of Economics, 13, 16-34.

Filiz, E. And E. OzBAy (2007): "Auctions with Anticipated Regret: Theory and Experiment," American Economic Review, 97, 1407-1418.

Garrett, T. And R. Sobel (1999): "Gamblers favor skewness, not risk: Further evidence from United States' lottery games," Economics Letters, 63, 85-90.

GeE, C. (2012): "A behavioral model of regret aversion," Unpublished manuscript.

Gilovich, T. And V. Medvec (1995): "The experience of regret: What, when, and why," Psychological Review, 102, 379-395.

Golec, J. And M. TAmarkin (1998): "Bettors love skewness, not risk, at the horse track," Journal of Political Economy, 106, 205-225.

Gollier, C. (1995): "The Comparative Statics of Changes in Risk Revisited," Journal of Economic Theory, 66, 522-536.

Gollier, C. AND B. SAlanié (2012): "Individual decisions under risk, risk sharing and asset prices with regret," Toulouse School of Economics.

Harvey, C. And A. Siddique (2000): "Conditional skewness in asset pricing tests," Journal of Finance, 55, 1263-1295.

Huang, R. J., A. Muermann, and L. Y. Tzeng (2014): "Regret and regulation," The Geneva Risk and Insurance Review, 39, 65-89.

Inman, J. J. AND L. MCAlister (1994): "Do coupon expiration dates affect consumer behavior?" Journal of Marketing Research, 16, 423-428.

Jullien, B. And B. Salanié (2000): "Extimating preferences under risk: The case of racetrack bettors," Journal of Political Economy, 108, 503-530. 
Kimball, M. (1990): "Precautionary savings in the small and in the large," Econometrica, $58,53-73$.

Loomes, G. (1988): "Further evidence of the impact of regret and disappointment in choice under uncertainty," Economica, 55.

Loomes, G. And R. Sugden (1982): "Regret theory: An alternative theory of rational choice under uncertainty," Economic Journal, 92, 805-824.

(1987a): "Some implications of a general form of regret theory," Journal of Economic Theory, 270-287.

(1987b): "Testing for regret and disappointment in choice under uncertainty," Economic Journal, 97, 118-129.

March, J. G. (1996): "Learning to be risk-averse," Psychological Review, 103, 309-319.

Michenaud, S. And B. Solnik (2008): "Applying regret theory to investment choices: Currency hedging decisions," Journal of International Money and Finance, 27, 677-694.

Muermann, A., O. Mitchell, And J. Volkman (2006): "Regret, portfolio choice, and guarantees in defined contribution schemes," Insurance: Mathematics and Economics, 39, $219-229$.

Quigain, J. (1994): "Regret theory with general choices," Journal of Risk and Uncertainty, 8, 153-165.

Rothschild, M. And J. Stiglitz (1970): "Increasing risk: I. A definition," Journal of Economic Theory, 2, 225-243.

$3,66-84$

SANDmO, A. (1971): "On the theory of the competitive firm under price uncertainty," American Economic Review, 61, 65-73.

SARVER, T. (2008): "Anticipating regret: Why fewer options may be better," Econometrica, $76,263-305$.

Savage, L. (1951): "The theory of statistical decision," Journal of the American Statistical Association, 46, 55-67.

Stalnaker, T., N. K. Cooch, and G. Schoenbaum (2015): "What the orbitofrontal cortex does not do," Nature Neuroscience, 18, 620-627.

Tchen, A. (1980): "Inequalities for distributions with given marginals," Annals of Probability, 8, 814-827.

Thaler, R. H. And W. T. Ziemba (1988): "Parimutuel betting markets: Racetracks and lotteries," Journal of Economic Perspectives, 2, 161-174. 
Tversky, A. And D. Kahneman (1992): "Advances in prospect theory: Cumulative representation of uncertainty," Journal of Risk and Uncertainty, 5, 297-323.

Wakker, P. (2010): Prospect Theory: For Risk and Ambiguity, Cambridge, UK: Cambridge University Press.

Wu, G. And R. Gonzalez (1996): "Curvature of the probability weighting function," Management Science, 42, 1676-1690.

Zeelenberg, M. And R. Pieters (2004): "Consequences of regret aversion in real life: The case of the Dutch postcode lottery," Organizational Behavior and Human Decision Processes, 93, 155-168. $3-18$.

(2007): "A theory of regret regulation 1.0," Journal of Consumer Psychology, 17, 